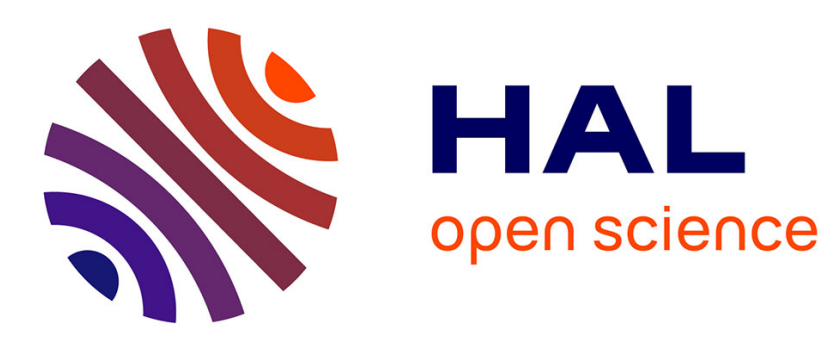

\title{
Specification and Safety Verification of Parametric Hierarchical Distributed Systems
}

\author{
Marius Bozga, Radu Iosif
}

\section{To cite this version:}

Marius Bozga, Radu Iosif. Specification and Safety Verification of Parametric Hierarchical Distributed Systems. Formal Aspects of Component Software, Oct 2021, Grenoble, France. hal-03418644

\section{HAL Id: hal-03418644 \\ https://hal.science/hal-03418644}

Submitted on 8 Nov 2021

HAL is a multi-disciplinary open access archive for the deposit and dissemination of scientific research documents, whether they are published or not. The documents may come from teaching and research institutions in France or abroad, or from public or private research centers.
L'archive ouverte pluridisciplinaire HAL, est destinée au dépôt et à la diffusion de documents scientifiques de niveau recherche, publiés ou non, émanant des établissements d'enseignement et de recherche français ou étrangers, des laboratoires publics ou privés. 


\title{
Specification and Safety Verification of Parametric Hierarchical Distributed Systems
}

\author{
Marius Bozga ${ }^{[0000-0003-4412-5684]}$ and Radu Iosif ${ }^{[0000-0003-3204-3294]}$ \\ Univ. Grenoble Alpes, CNRS, Grenoble INP*^, VERIMAG, 38000 Grenoble, France \\ \{marius.bozga, radu.iosif\} @univ-grenoble-alpes.fr
}

\begin{abstract}
We introduce a term algebra as a new formal specification language for the coordinating architectures of distributed systems consisting of a finite yet unbounded number of components. The language allows to describe infinite sets of systems whose coordination between components share the same pattern, using inductive definitions similar to the ones used to describe algebraic data types or recursive data structures. Further, we give a verification method for the parametric systems described in this language, relying on the automatic synthesis of structural invariants that enable proving general safety properties (absence of deadlocks and critical section violations). The invariants are defined using the WS $\kappa S$ fragment of the monadic second order logic, known to be decidable by a classical automata-logic connection. This reduces the safety verification problem to checking satisfiability of a WS $\kappa S$ formula. We implemented the invariant synthesis method into a prototype tool and carried out verification experiments on a number of non-trivial models specified using the term algebra.
\end{abstract}

\section{Introduction}

The separation between behavior and coordination is a fundamental principle in the design of large-scale distributed systems [16. By behavior we mean a set of traces of observable events. A component is a representation of a behavior, by means of a (finite) state machine, whose actions are labeled by events. The architecture of the system defines the interactions between components, as sets of events that must occur simultaneously in several components. For instance, Fig. 13 shows a token-ring systems, whose components are depicted in yellow boxes (behaviors are modeled by the finite-state machines within the boxes) and whose architecture is the set of connections between components (depicted with solid lines). Such high-level models of real-life distributed systems are suitable for reasoning about correctness in the early stages of system design, when details related to network reliability or the implementation of coordination mechanisms, by means of low-level synchronization mechanisms (e.g. semaphores, monitors, compare-and-swap, etc.) are abstracted away.

${ }_{\star \star \star}$ Institute of Engineering Univ. Grenoble Alpes 
This modular view of a distributed system is key to scalable design methods that exploit a conceptual hierarchy, in which each module is split into submodules. For instance, a ring is a chain whose final output port is connected to the initial input port, whereas a chain consists of a (head) component linked to a separate (tail) chain (Fig. 1b). Furthermore, system designers are accustomed to the use of predefined architectural patterns, that define the interactions between (unboundedly large) sets of modules (e.g. crowds, rings, pipelines, stars, trees, etc.). In this context, the contribution of the paper is three-fold.

1. We introduce a formal language to describe the coordinating architectures of distributed systems parameterized by (i) the number of components of each type that are active in the system, e.g. a system with $n$ readers and $m$ writers, in which $n$ and $m$ are not known a priori and (ii) the pattern in which the interactions occur (e.g. a pipeline, ring, star or more general hypergraph structures). The language uses predicate symbols to hierarchically break the architecture into sub-modules. The interpretation of these predicate symbols is defined inductively by rewriting rules consisting of terms that contain predicate atoms, in a way that recalls the usual definitions of algebraic datatypes [2] or heaps [18.

2. We tackle the parametric safety problem for systems described in this language, which is checking that the reachable states of every jnstance stays clear of a set of global error configurations, such as deadlocks or critical section violations. We synthesize invariants directly from the syntactic description of the system, generate WS $\kappa$ S formulæ [19] that are unsatisfiable only if every system described by the given inductive definitions is safe and use off-the-shelf WS $\kappa S$ solvers 11] for proving safety automatically. The invariant synthesis method models the set of executions of a parametric system as a boolean (1-safe) Petri net of unbounded size and computes structural invariants (trap invariants, linear invariants) of this Petri net.

3. We implemented the invariant synthesis in a prototype tool and experimented with a number of parametric component-based systems with non-trivial architectural patterns, such as trees with root links, trees with linked leaves, token-rings with or without a main controller, etc.

Example 1. Let us consider a distributed system consising of components of type C, having two interaction ports, namely in and out and whose behavior is described by a finite state machine with transitions $q_{0} \stackrel{\text { out }}{\longrightarrow} q_{1}$ and $q_{1} \stackrel{\text { in }}{\longrightarrow} q_{0}$. These components are arranged in a ring, such that the out port of a component is connected to the in port of its right neighbour, with the exception of the last component, whose out port connects to the in port of the first component (Fig. 17). The connections (interactions) in the system are described by the predicate Ring(), defined inductively by the rules below:

$$
\begin{aligned}
\operatorname{Ring}() & \leftarrow \nu y_{1} \nu y_{2} \cdot\left\langle\operatorname{out}\left(y_{2}\right) \cdot \operatorname{in}\left(y_{1}\right)\right\rangle\left(\operatorname{Chain}\left(y_{1}, y_{2}\right)\right) \\
\operatorname{Chain}\left(x_{1}, x_{2}\right) & \leftarrow\left\langle\operatorname{out}\left(x_{1}\right) \cdot \operatorname{in}\left(x_{2}\right)\right\rangle\left(\operatorname{Comp}\left(x_{1}\right), \operatorname{Comp}\left(x_{2}\right)\right) \\
\operatorname{Chain}\left(x_{1}, x_{2}\right) & \leftarrow \nu y_{1} \cdot\left\langle\operatorname{out}\left(x_{1}\right) \cdot \operatorname{in}\left(y_{1}\right)\right\rangle\left(\operatorname{Comp}\left(x_{1}\right), \operatorname{Chain}\left(y_{1}, x_{2}\right)\right) \\
\operatorname{Comp}(x) & \leftarrow \mathrm{C}(x)
\end{aligned}
$$




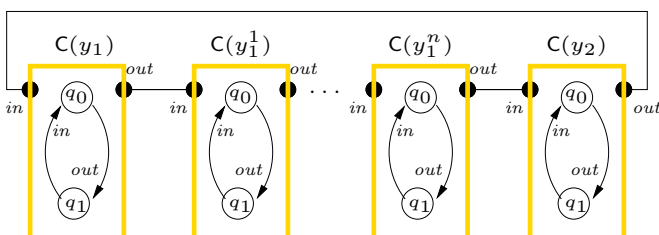

(a)

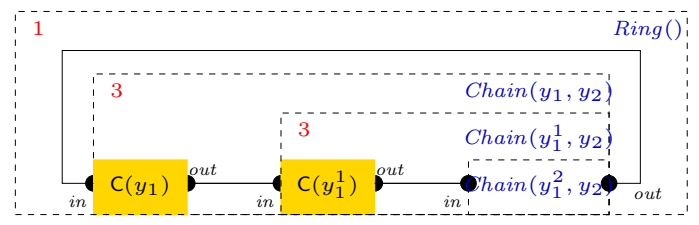

(b)

Fig. 1. Recursive Specification of a Token-Ring System

Rule (1) states that a $\operatorname{Ring}()$ consist of a $\operatorname{Chain}\left(y_{1}, y_{2}\right)$, where $y_{1}$ and $y_{2}$ are the indices of the first and last component: 1 , respectively. The last out port is connected to the first in port, written as out $\left(y_{2}\right) \cdot \operatorname{in}\left(y_{1}\right)$. Rule $(2)$ states that the least Chain $\left(x_{1}, x_{2}\right)$ consists of two instances of type $\mathrm{C}$, namely $\mathrm{C}\left(x_{1}\right)$ and $\mathrm{C}\left(x_{2}\right)$, and the out port of $x_{1}$ connects to the in port of $x_{2}$, described as out $\left(x_{1}\right) \cdot \operatorname{in}\left(x_{2}\right)$. Rule (3) gives the inductive step, namely that every Chain $\left(x_{1}, x_{2}\right)$ consists of a component $\mathrm{C}\left(x_{1}\right)$ that interacts with a disjoint chain from $y_{1}$ to $x_{2}$. Here the binder $\nu y_{1}$ makes sure the value of $y_{1}$ is different from the value of every other variable in the system. Since this binder is used in a recursive rule, each identifier in a subsequent unfolding of Chain $\left(y_{1}, x_{2}\right)$ is guaranteed to be unique. Last, rule (4) is used to instantiate (i.e. create new) components of type C. In principle, this rule is not necessary, as any occurrence of a predicate $\operatorname{Comp}(y)$ can be replaced by a component $\mathrm{C}(y)$, however it is considered for technical reasons related to our invariant-based verification approach.

Fig. 1p shows the unfoldings of this set of recursive definitions. The system depicted in Fig. 17 is obtained by an application of rule (1), followed by $n$ applications of rule (3), ending with an application of rule (2). The first two applications of (3) following the application of (1) are depicted in Fig. 1b, with rule labels annotated (each application of rule (3) creates a fresh variable, denoted by $y_{1}^{1}, \ldots, y_{1}^{n}$, respectively).

\section{Preliminaries}

This section introduces preliminary definitions used throughout the paper. Given sets $A$ and $B$, we denote by $A \rightarrow B$ the set of total functions $f: A \rightarrow B$. If $\mathbf{a}=\left\langle a_{1}, \ldots, a_{n}\right\rangle$ is a tuple of values from $A$, then $f(\mathbf{a}) \stackrel{\text { def }}{=}\left\langle f\left(a_{1}\right), \ldots, f\left(a_{n}\right)\right\rangle$. By $\mathbf{a} \cdot \mathbf{b}$ we denote the concatenation of tuples $\mathbf{a}$ and $\mathbf{b}$. For two positive integers $k, \ell \in \mathbb{N}$, we denote by $[k, \ell]$ the set $\{k, k+1, \ldots, \ell\}$, assumed to be empty if $k>\ell$. The cardinality of a finite set $A$ is denoted by $|A|$.

Trees Trees play a key role in the definition of parametric distributed systems from the following section (\$3). Let $\kappa \geq 1$ be an integer constant, fixed throughout this paper, and let $[1, \kappa]^{*}$ denote the set of finite sequences of integers between

\footnotetext{
${ }^{1}$ First and last are understood here in the order of unfolding of the rewriting rules.
} 
1 and $\kappa$, called nodes in the following. A $\kappa$-ary tree $\mathcal{T}$ is a partial function mapping $[1, \kappa]^{*}$ to a set of labels. The domain of $\mathcal{T}$, denoted nodes $(\mathcal{T})$, is such that $w i \in \operatorname{nodes}(\mathcal{T})$ for some $i \in[1, \kappa]$ only if $w \in \operatorname{nodes}(\mathcal{T})$ and $w j \in \operatorname{nodes}(\mathcal{T})$ for all $j \in[1, i-1]$. The root of $\mathcal{T}$ is the empty sequence $\epsilon$, the children of a node $w \in \operatorname{nodes}(\mathcal{T})$ are $\{w i \in \operatorname{nodes}(\mathcal{T}) \mid i \in[1, \kappa]\}$ and the parent of a node $w i, i \in$ $[1, \kappa]$, is $w$. The leaves of $\mathcal{T}$ are leaves $(\mathcal{T}) \stackrel{\text { def }}{=}\{w \in \operatorname{nodes}(\mathcal{T}) \mid w .1 \notin \operatorname{nodes}(\mathcal{T})\}$. The subtree of $\mathcal{T}$ rooted at $w$ is defined by $\operatorname{nodes}\left(\mathcal{T} \downarrow_{w}\right) \stackrel{\text { def }}{=}\left\{w^{\prime} \mid w w^{\prime} \in \operatorname{nodes}(\mathcal{T})\right\}$ and $\mathcal{T} \downarrow_{w}\left(w^{\prime}\right) \stackrel{\text { def }}{=} \mathcal{T}\left(w w^{\prime}\right)$, for all $w^{\prime} \in \operatorname{nodes}\left(\mathcal{T} \downarrow_{w}\right)$.

The invariant synthesis method uses the restriction of monadic second order logic to trees of branching degree $\kappa$ and quantification over finite sets only. Let $\mathbb{V}_{1}=\{x, y, z, \ldots\}$ and $\mathbb{V}_{2}=\{X, Y, Z, \ldots\}$ be countably infinite sets of first and second order variables, respectively. The formulæ of the WS $\kappa S$ logic are defined inductively by the syntax:

$$
\begin{array}{lr}
\tau::=\epsilon\left|x \in \mathbb{V}_{1}\right| \tau_{1} . i, i \in[1, \kappa] & \text { terms } \\
\phi::=\tau=\tau|X(\tau)| \phi \wedge \phi|\neg \phi| \exists x . \phi \mid \exists X . \phi & \text { formulæ }
\end{array}
$$

As usual, we write $\phi_{1} \vee \phi_{2} \stackrel{\text { def }}{=} \neg\left(\neg \phi_{1} \wedge \neg \phi_{2}\right), \phi_{1} \rightarrow \phi_{2} \stackrel{\text { def }}{=} \neg \phi_{1} \vee \phi_{2}, \phi_{1} \leftrightarrow \phi_{2} \stackrel{\text { def }}{=}$ $\left(\phi_{1} \rightarrow \phi_{2}\right) \wedge\left(\phi_{2} \rightarrow \phi_{1}\right), \forall x . \phi \stackrel{\text { def }}{=} \neg \exists x . \neg \phi$ and $\forall X . \phi \stackrel{\text { def }}{=} \neg \exists X . \neg \phi$. WS $\kappa S$ formulæ are interpreted over an infinite tree, where first order variables $x \in \mathbb{V}_{1}$ range over individual nodes $n \in[1, \kappa]^{*}$, second order variables $X \in \mathbb{V}_{2}$ range over finite sets of nodes, $\epsilon$ is a constant symbol interpreted as the root of the tree and, for all $i \in[1, \kappa]$, the notation $i$ is is interpreted as the function mapping each $w \in[1, \kappa]^{*}$ into $w i$. Given a valuation $\nu: \mathbb{V}_{1} \cup \mathbb{V}_{2} \rightarrow[1, \kappa]^{*} \cup 2^{[1, \kappa]^{*}}$, such that $\nu(x) \in[1, \kappa]^{*}$, for each $x \in \mathbb{V}_{1}$ and $\nu(X) \subseteq[1, \kappa]^{*}(\nu(X)$ is finite), for each $X \in \mathbb{V}_{2}$, the satisfaction relation $\models$ is defined inductively, as usual [14. A valuation $\nu$ is a model of a formula $\phi$ iff $\nu \models \phi$. A formula is satisfiable if and only if it has a model.

Petri nets A Petri net (PN) is a tuple $\mathrm{N}=\langle S, T, E\rangle$, where $S$ is a set of places, $T$ is a set of transitions, $S \cap T=\emptyset$, and $E \subseteq(S \times T) \cup(T \times S)$ is a set of edges. Given $x, y \in S \cup T$, we write $E(x, y) \stackrel{\text { def }}{=} 1$ if $(x, y) \in E$ and $E(x, y) \stackrel{\text { def }}{=} 0$, otherwise. Let $\bullet \stackrel{\text { def }}{=}\{y \in S \cup T \mid E(y, x)=1\}, x \stackrel{\text { def }}{=}\{y \in S \cup T \mid E(x, y)=1\}$ and lift these definitions to sets of nodes. A marking of $\mathrm{N}$ is a function $\mathrm{m}: S \rightarrow \mathbb{N}$. A transition $t$ is enabled in $\mathrm{m}$ if and only if $\mathrm{m}(s)>0$ for each place $s \in \bullet$. We write $\mathrm{m} \stackrel{t}{\rightarrow} \mathrm{m}^{\prime}$ whenever $t$ is enabled in $\mathrm{m}$ and $\mathrm{m}^{\prime}(s)=\mathrm{m}(s)-E(s, t)+E(t, s)$, for all $s \in S$ and $t \in T$. A sequence of transitions $\sigma=t_{1}, \ldots, t_{n}$ is a firing sequence, written $\mathrm{m} \stackrel{\sigma}{\rightarrow} \mathrm{m}^{\prime}$ if and only if either (i) $n=0$ and $\mathrm{m}=\mathrm{m}^{\prime}$, or (ii) $n \geq 1$ and there exist markings $\mathrm{m}_{1}, \ldots, \mathrm{m}_{n-1}$ such that $\mathrm{m} \stackrel{t_{1}}{\longrightarrow} \mathrm{m}_{1} \ldots \mathrm{m}_{n-1} \stackrel{t_{n}}{\longrightarrow} \mathrm{m}^{\prime}$.

A marked Petri net is a pair $\mathcal{N}=\left(\mathrm{N}, \mathrm{m}_{0}\right)$, where $\mathrm{m}_{0}$ is the initial marking of $\mathrm{N}$. A marking $\mathrm{m}$ is reachable in $\mathcal{N}$ if there exists a firing sequence $\sigma$ such that $\mathrm{m}_{0} \stackrel{\sigma}{\rightarrow} \mathrm{m}$. We denote by $\mathcal{R}(\mathcal{N})$ the set of reachable markings of $\mathcal{N}$. A marked PN $\mathcal{N}$ is boolean if $\mathrm{m}(s) \leq 1$, for each $s \in S$ and $\mathrm{m} \in \mathcal{R}(\mathcal{N})$. All marked PNs considered in the following will be boolean and we shall silently blur the distinction between a marking $\mathrm{m}: S \rightarrow\{0,1\}$ and the set $\{s \in S \mid \mathrm{m}(s)=1\}$.

Given a set of markings $\mathcal{E}$, a marked $\mathrm{PN} \mathcal{N}$ is safe w.r.t. $\mathcal{E}$ if and only if $\mathcal{R}(\mathcal{N}) \cap \mathcal{E}=\emptyset$. A set of markings $\mathcal{M}$ is an inductive invariant of $\mathcal{N}=\left(\mathrm{N}, \mathrm{m}_{0}\right)$ if 
and only if $\mathrm{m}_{0} \in \mathcal{M}$ and for each $\mathrm{m} \stackrel{t}{\rightarrow} \mathrm{m}^{\prime}$ such that $\mathrm{m} \in \mathcal{M}$, we have $\mathrm{m}^{\prime} \in \mathcal{M}$. It is known that $\mathcal{R}(\mathcal{N})$ is the least inductive invariant of $\mathcal{N}$, thus $\mathcal{N}$ is safe w.r.t $\mathcal{E}$ if it has an inductive invariant $\mathcal{M}$ disjoint from $\mathcal{E}$.

Components In this paper we are concerned with systems consisting of an unbounded number of components that are replicas of a fairly small set of patterns, called component types. Let $\mathbb{P}=\{a, b, \ldots\}$ and $\mathbb{S}=\{s, t, \ldots\}$ be countably infinite sets of ports and states, respectively. An injective function $P$ (resp. $S$ ) mapping tree nodes to ports (resp. states) is called a port type (resp. state type). A component type is a tuple $\mathcal{B}=\langle\mathcal{P}, \mathcal{S}, \mathcal{I}, \Delta\rangle$, where $\mathcal{P} \subseteq[1, \kappa]^{*} \rightarrow \mathbb{P}$ and $\mathcal{S} \subseteq[1, \kappa]^{*} \rightarrow \mathbb{S}$ are finite sets of port and state types, $\mathcal{I} \in \mathcal{S}$ is the initial state type, and $\Delta$ is a finite set of transition rules $S \stackrel{P}{\rightarrow} T$, where $S, T \in \mathcal{S}$ and $P \in \mathcal{P}$. In addition, we require that (i) the elements of $\mathcal{P}$ (resp. $\mathcal{S}$ ) have pairwise disjoint ranges and (ii) for any two transition rules $S_{1} \stackrel{P_{1}}{\longrightarrow} S_{1}^{\prime}, S_{2} \stackrel{P_{2}}{\longrightarrow} S_{2}^{\prime}$, if $P_{1}=P_{2}$ then $S_{1}=S_{2}$ and $S_{1}^{\prime}=S_{2}^{\prime}$. For a transition rule $S \stackrel{P}{\rightarrow} S^{\prime} \in \Delta$, let $P^{\stackrel{\text { def }}{=}} S$ and $P^{\bullet} \stackrel{\text { def }}{=} S^{\prime}$ denote the pre- and post-state type of the unique transition rule whose label is the port type $P$.

The replicas of a component type are indexed (distinguished) by tree nodes ${ }^{2}$ Given a component type $\mathcal{B}=\langle\mathcal{P}, \mathcal{S}, \mathcal{I}, \Delta\rangle$ and a tree node $w \in[1, \kappa]^{*}$, we define the component $\mathcal{B}(w) \stackrel{\text { def }}{=}\langle\{P(w) \mid P \in \mathcal{P}\},\{S(w) \mid S \in \mathcal{S}\}, \mathcal{I}(w),\{S(w) \stackrel{P(w)}{\longrightarrow}$ $\left.\left.S^{\prime}(w) \mid S \stackrel{P}{\rightarrow} S^{\prime} \in \Delta\right\}\right\rangle$. Note that the sets of ports $\{P(w) \mid P \in \mathcal{P}\}$ (resp. states $\{S(w) \mid S \in \mathcal{S}\}$ ) of different replicas of the same component type are disjoint, because the port (state) types are required to have disjoint ranges. We slightly abuse notation by writing $\bullet^{\bullet}(P(w)) \stackrel{\text { def }}{=}(P)(w)$ and $(P(w))^{\bullet} \stackrel{\text { def }}{=}(P)^{\bullet}(w)$ (we omit brackets when they are clear from the context). We consider below a set $\mathbb{B}$ of component types, with pairwise disjoint sets of port and state types.

Architectures The coordination in a system is defined by architectures. An interaction $\pi \in 2^{\mathbb{P}}$ is a finite set of ports. An architecture $\gamma \subseteq 2^{\mathbb{P}}$ is a finite set of interactions. Given component types $\mathcal{B}_{i}=\left\langle\mathcal{P}_{i}, \mathcal{S}_{i}, \mathcal{I}_{i}, \Delta_{i}\right\rangle \in \mathbb{B}$ and tree nodes $w_{i} \in[1, \kappa]^{*}$, the behavior of the system consisting of the components $\mathcal{B}_{i}\left(w_{i}\right)$, $i=1, \ldots, n$, coordinated by the architecture $\gamma$ is defined by the marked PN $\gamma\left(\mathcal{B}_{1}\left(w_{1}\right), \ldots, \mathcal{B}_{n}\left(w_{n}\right)\right) \stackrel{\text { def }}{=}\left(\langle S, \gamma, E\rangle, \mathrm{m}_{0}\right)$, where $S \stackrel{\text { def }}{=} \bigcup_{i=1}^{n}\left\{S\left(w_{i}\right) \mid S \in \mathcal{S}_{i}\right\}$ is the set of places, for each interaction $\pi \in \gamma$, the edges to (from) $\pi$ are given by - $\pi \stackrel{\text { def }}{=}\{\bullet p \mid p \in \pi\}\left(\pi^{\bullet} \stackrel{\text { def }}{=}\left\{p^{\bullet} \mid p \in \pi\right\}\right)$ and the initial marking is $\mathrm{m}_{0} \stackrel{\text { def }}{=}\left\{\mathcal{I}_{i}\left(w_{i}\right) \mid\right.$ $i \in[1, n]\}$.

Example 2. Fig. 2 shows the marked PN that defines the behavior of the system from Fig. 1 1 . The tree node $1 \ldots 1$ ( $i$ times) is represented by its value $i$ in the unary encoding. The interaction $\{\operatorname{in}(n)$, out(1) $\}$ is duplicated, for readability. The initially marked places are surrounded by dashed circles.

\footnotetext{
${ }^{2}$ We identify components by tree nodes in preparation of the ground for the verification technique from \$4 However, these definitions can be given in general, for any countably infinite set of identifiers.
} 


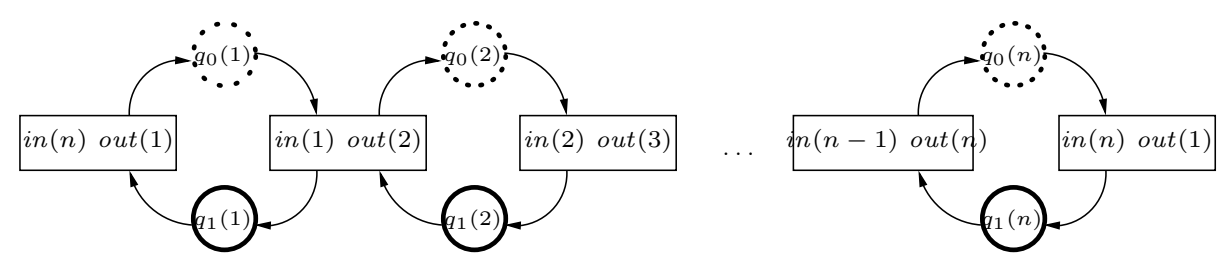

Fig. 2. The Behavior of a Token-Ring System

\section{A Term Algebra of Behaviors}

In this section we introduce a term algebra for describing the systems resulting from the application of an architecture to an unbounded number of component type instances (see Example1 for the specification of a token-ring system in this language). Let $\mathbb{A}$ be a countably infinite set of predicate symbols, and let $\#(A)$ denote the arity of the predicate symbol $A \in \mathbb{A}$.

Syntax The following syntax generates behavioral terms inductively:

$$
\begin{array}{ll}
P \in[1, \kappa]^{*} \mapsto \mathbb{P}, x \in \mathbb{V}_{1}, \mathcal{B} \in \mathbb{B}, \mathrm{A} \in \mathbb{A} & \\
I::=P(x) \mid I_{1} \cdot I_{2} & \text { interactions } \\
\Gamma::=I \mid \Gamma_{1}+\Gamma_{2} & \text { architectures } \\
\mathrm{b}::=\mathcal{B}(x)\left|\langle\Gamma\rangle\left(\mathrm{b}_{1}, \ldots, \mathrm{b}_{n}\right)\right| \nu x . \mathrm{b}_{1} \mid \mathrm{A}\left(x_{1}, \ldots, x_{\#(\mathrm{~A})}\right) & \text { behavioral terms }
\end{array}
$$

A variable $x$ occurring in a behavioral term $\mathrm{b}$ is free if it does not occur in the scope of some subterm of the form $\nu x \cdot b_{1}$ and bound otherwise. The set of free variables occurring in a term $b$ is denoted by $\mathrm{fv}(\mathrm{b})$. In the following, we assume that all bound variables occurring in a term are pairwise distinct and distinct from the free variables. This assumption loses no generality because terms obtained by $\alpha$-conversion (renaming of bound variables) are usually viewed as the same term. A term $\mathrm{b}$ is closed if $\mathrm{fv}(\mathrm{b})=\emptyset$ and predicateless if no predicates from $\mathbb{A}$ occur in $\mathrm{b}$. We denote by $\mathrm{b}\left[y_{1} / x_{1}, \ldots, y_{n} / x_{n}\right]$ the term obtained by substituting the variable $x_{i} \in \mathrm{fv}$ (b) with $y_{i}$, for each $i \in[1, n]$. We write size(b) for the number of occurrences of symbols in $b$.

A term $\mathcal{B}(x)$ is called an instance atom and a term $\mathrm{A}\left(x_{1}, \ldots, x_{n}\right)$ is called a predicate atom. We denote by $\#_{\text {pred }}(\mathrm{b})$ the number of occurrences of predicate atoms and by $\operatorname{pred}_{i}(\mathrm{~b})$ the predicate atom that occurs $i$-th in b, for $i \in$ $\left[1, \#_{\text {pred }}(b)\right]$, in some linear order of the nodes in the syntax tree of $b$. The predicate symbols are interpreted as the least sets of predicateless terms inductively defined by a rewriting system:

Definition 1. A rewriting system is a finite set $\mathcal{R}$ of rules of one of the forms:

$$
\begin{aligned}
& \mathrm{A}(x) \leftarrow \mathcal{B}(x) \\
& \mathrm{A}\left(x_{1}, \ldots, x_{\#(\mathrm{~A})}\right) \leftarrow \nu y_{1} \ldots \nu y_{n} .\langle\Gamma\rangle\left(\mathrm{A}_{1}\left(z_{1}^{1}, \ldots, z_{\#\left(\mathrm{~A}_{1}\right)}^{1}\right), \ldots, \mathrm{A}_{m}\left(z_{1}^{m}, \ldots, z_{\#\left(\mathrm{~A}_{m}\right)}^{m}\right)\right) \\
& \text { where } m \geq 1 \text { and }\left\{\left\{z_{1}^{i}, \ldots, z_{\#\left(\mathrm{~A}_{i}\right)}^{i}\right\}\right\}_{i=1}^{m} \text { is a partition of }\left\{x_{1}, \ldots, x_{\#(\mathrm{~A})}, y_{1}, \ldots, y_{n}\right\}
\end{aligned}
$$

For instance, in Example 1) rule (4) is an instantiation rule, whereas (1), 2) and (3) are inductive rules. We write $\mathrm{A}\left(x_{1}, \ldots, x_{\#(\mathrm{~A})}\right) \leftarrow_{\mathcal{R}}$ b for $\mathrm{A}\left(x_{1}, \ldots, x_{\#(\mathrm{~A})}\right) \leftarrow$ 
$\mathrm{b} \in \mathcal{R}$. The size of $\mathcal{R}$ is $\operatorname{size}(\mathcal{R}) \stackrel{\text { def }}{=} \sum_{\mathrm{A}\left(x_{1}, \ldots, x_{\#(\mathrm{~A})}\right) \leftarrow_{\mathcal{R}} \mathrm{b}} \operatorname{size}(\mathrm{b})$. Given behavioral terms $b_{1}$ and $b_{2}$, we denote by $b_{1} \stackrel{r}{\Leftarrow} b_{2}$ the rewriting step that obtains $b_{2}$ by replacing a predicate atom $\mathrm{A}\left(y_{1}, \ldots, y_{\#(\mathrm{~A})}\right)$ in $\mathrm{b}_{1}$ with $\mathrm{b}\left[y_{1} / x_{1}, \ldots, y_{\#(\mathbf{A})} / x_{\#(\mathbf{A})}\right]$, where $\mathrm{r}=\left(\mathrm{A}\left(x_{1}, \ldots, x_{\# \mathrm{~A}}\right) \leftarrow \mathrm{b}\right)$ is a rule of $\mathcal{R}$ and all bound variables in $\mathrm{b}$ are renamed to avoid clashes with the variables from $b_{1}$. We write $[b]_{\mathcal{R}}$ for the set of predicateless terms obtained from $b$ by exhaustively applying the rewriting rules from $\mathcal{R}$ to it.

Semantics Let us consider a given closed behavioral term $b$ and a rewriting system $\mathcal{R}$. First, we define the semantics of a (closed) predicateless behavioral term $\mathrm{t} \in[\mathrm{b}]_{\mathcal{R}}$, as the behavior (i.e. marked PN) resulting from joining the components defined by the instance atoms from $t$, via the architecture consisting of all the interactions that occur in t. This definition is done in two steps:

(a) we write $\mathrm{t}$ in prenex form as $\nu x_{1} \ldots \nu x_{n} . \mathrm{u}$, where $\mathrm{u}$ contains no more terms of the form $\nu x . \mathrm{b}$, by moving all the $\nu$ binders upfront. Because all bound variables in $t$, including those introduced by rewriting, are assumed to be pairwise distinct, this step incurs no name clashes.

(b) we apply the following flattening relation exhaustively:

$$
\left\langle\Gamma_{1}\right\rangle\left(\left\langle\Gamma_{2}\right\rangle\left(\mathrm{b}_{1}, \ldots \mathrm{b}_{i}\right), \mathrm{b}_{i+1}, \ldots \mathrm{b}_{n}\right) \rightsquigarrow\left\langle\Gamma_{1}+\Gamma_{2}\right\rangle\left(\mathrm{b}_{1}, \ldots, \mathrm{b}_{n}\right)
$$

Example 3. Consider the below rewriting sequence, using rules from Example 1:

$$
\begin{aligned}
& \operatorname{Ring}() \Leftarrow \nu y_{1} \nu y_{2} \cdot\left\langle\operatorname{out}\left(y_{2}\right) \cdot \operatorname{in}\left(y_{1}\right)\right\rangle\left(\operatorname{Chain}\left(y_{1}, y_{2}\right)\right) \\
& \Leftarrow \nu y_{1} \nu y_{2} \cdot\left\langle\operatorname{out}\left(y_{2}\right) \cdot \operatorname{in}\left(y_{1}\right)\right\rangle\left(\nu y_{1}^{1} \cdot\left\langle\operatorname{out}\left(y_{1}\right) \cdot \operatorname{in}\left(y_{1}^{1}\right)\right\rangle\left(\operatorname{Comp}\left(y_{1}\right), \operatorname{Chain}\left(y_{1}^{1}, y_{2}\right)\right)\right) \\
& \Leftarrow \nu y_{1} \nu y_{2} \cdot\left\langle\operatorname{out}\left(y_{2}\right) \cdot \operatorname{in}\left(y_{1}\right)\right\rangle\left(\nu y _ { 1 } ^ { 1 } \cdot \langle \operatorname { o u t } ( y _ { 1 } ) \cdot \operatorname { i n } ( y _ { 1 } ^ { 1 } ) \rangle \left(\operatorname{Comp}\left(y_{1}\right),\right.\right. \\
& \left.\left.\left\langle\operatorname{out}\left(y_{1}^{1}\right) \cdot \operatorname{in}\left(y_{2}\right)\right\rangle\left(\operatorname{Comp}\left(y_{1}^{1}\right), \operatorname{Comp}\left(y_{2}\right)\right)\right)\right) \\
& \Leftarrow \ldots \Leftarrow \nu y_{1} \nu y_{2} \cdot\left\langle\operatorname{out}\left(y_{2}\right) \cdot \operatorname{in}\left(y_{1}\right)\right\rangle\left(\nu y _ { 1 } ^ { 1 } \cdot \langle \operatorname { o u t } ( y _ { 1 } ) \cdot \operatorname { i n } ( y _ { 1 } ^ { 1 } ) \rangle \left(\mathrm{C}\left(y_{1}\right),\right.\right. \\
& \left.\left.\left\langle\operatorname{out}\left(y_{1}^{1}\right) \cdot \operatorname{in}\left(y_{2}\right)\right\rangle\left(\mathrm{C}\left(y_{1}^{1}\right), \mathrm{C}\left(y_{2}\right)\right)\right)\right)=\mathrm{t}
\end{aligned}
$$

By applying flattening to the last term, we obtain:

$\mathbf{t}^{\aleph \aleph}=\nu y_{1} \nu y_{2} \cdot\left\langle\operatorname{out}\left(y_{2}\right) \cdot \operatorname{in}\left(y_{1}\right)+\operatorname{out}\left(y_{1}\right) \cdot \operatorname{in}\left(y_{1}^{1}\right)+\operatorname{out}\left(y_{1}^{1}\right) \cdot \operatorname{in}\left(y_{2}\right)\right\rangle\left(\mathrm{C}\left(y_{1}\right), \mathrm{C}\left(y_{1}^{1}\right), \mathrm{C}\left(y_{2}\right)\right)$

Note that every chain $t_{1} \rightsquigarrow t_{2} \rightsquigarrow \ldots$ is finite, because the height of terms strictly decreases with flattening. The result of flattening is of the form $\mathrm{t} \leadsto \stackrel{\text { def }}{=}$ $\langle\Gamma\rangle\left(\mathcal{B}_{1}\left(x_{1}\right), \ldots, \mathcal{B}_{n}\left(x_{n}\right)\right)$, where $\Gamma=\sum_{k=1}^{m} P_{k 1}\left(x_{k 1}\right) \cdot \ldots \cdot P_{k r_{k}}\left(x_{k r_{k}}\right)$ is an architecture description, such that each $P_{k \ell} \in[1, \kappa]^{*} \mapsto \mathbb{P}$ is a port type, $x_{k \ell} \in$ $\left\{x_{1}, \ldots, x_{n}\right\}$, for all $k \in[1, m]$ and $\ell \in\left[1, r_{k}\right]$. Given an injective valuation $\nu: \mathbb{V}_{1} \rightarrow[1, \kappa]^{*}$ that maps variables into distinct nodes of a tree of branching degree $\kappa$, we define the architecture $\Gamma(\nu) \stackrel{\text { def }}{=}\left\{\left\{P_{k 1}\left(\nu\left(x_{k 1}\right)\right), \ldots, P_{k r_{k}}\left(\nu\left(x_{k r_{k}}\right)\right)\right\} \mid\right.$ $k \in[1, m]\}$ and the behavior:

$$
\mathrm{B}_{\nu}^{\mathrm{t}} \stackrel{\text { def }}{=} \Gamma(\nu)\left(\mathcal{B}_{1}\left(\nu\left(x_{1}\right)\right), \ldots, \mathcal{B}_{n}\left(\nu\left(x_{n}\right)\right)\right)
$$

The semantics of the behavioral term $\mathrm{b}$ in the rewriting system $\mathcal{R}$ is the following set of marked PNs:

$$
\llbracket \mathrm{b} \rrbracket_{\mathcal{R}} \stackrel{\text { def }}{=}\left\{\mathrm{B}_{\nu}^{\mathrm{t}} \mid \mathrm{t} \in[\mathrm{b}]_{\mathcal{R}}, \nu \in \mathbb{V}_{1} \rightarrow[1, \kappa]^{*} \text { injective }\right\}
$$

As a remark, the flattening step is required because applying an architecture to a set of components is a global operation; if an interaction $P_{k 1}\left(x_{k 1}\right) \cdot \ldots$. $P_{k r_{k}}\left(x_{k r_{k}}\right)$ occurs as a monomial in the architecture description $\Gamma$ of a subterm 
$\mathrm{u}=\langle\Gamma\rangle\left(\mathrm{t}_{1}, \ldots, \mathrm{t}_{\ell}\right)$ of $\mathrm{t}$ and some variable $x_{k i}$ occurs in an instance atom $\mathcal{B}\left(x_{k i}\right)$ in $\mathrm{t}$ but not in $\mathrm{u}$, the interaction would be ignored if we applied $\Gamma(\nu)$ directly to $\mathrm{B}_{\nu}^{\mathrm{t}_{1}}, \ldots,, \mathrm{B}_{\nu}^{\mathrm{t}_{\ell}}$, for some injective valuation $\nu$.

\section{The Parametric Safety Problem}

Having defined a rewriting-based term algebra for the specification of distributed systems, we move on to the problem of verifying that every behavior generated by a given rewriting system $\mathcal{R}$, starting from a given behavioral term $b$ is safe with respect to a given set of error markings. This problem is challenging, because we ask for a proof of safety that holds for the behavior(s) of every predicateless rewriting of the behavioral term, i.e. for each $t \in[b]_{\mathcal{R}}$. Since, even for token-ring systems with finite-state components, the parametric safety problem is undecidable [9], we resort to a sound but necessarily incomplete solution, that consists in computing inductive invariants.

Structural invariants In contrast with the classical approach to invariant synthesis, based on a fixpoint iteration in an abstract domain [8], we consider a particular class of invariants, that can be obtained directly from the syntactic structure of the marked PN representation of behaviors. For this reason, we call these invariants structural. In the following, we define two kinds of such invariants, namely trap and mutex invariants:

Definition 2. Given a marked $P N \mathcal{N}=\left(\langle S, T, E\rangle, \mathrm{m}_{0}\right)$, a set $\theta \subseteq S$ is a:

1. trap if $\left|\theta \cap \mathrm{m}_{0}\right| \geq 1$ and, for any $t \in T$, if $|\theta \cap \bullet t| \geq 1$ then $\left|\theta \cap t^{\bullet}\right| \geq 1$.

2. mutex if $\left|\theta \cap \mathrm{m}_{0}\right|=1$ and, for any $t \in T$, we have $|\theta \cap \bullet|=\left|\theta \cap t^{\bullet}\right| \leq 1$.

The structural invariants of $\mathcal{N}$ are the trap and mutex invariants, repectively:

A. $\Theta(\mathcal{N}) \stackrel{\text { def }}{=}\{\mathrm{m}$ marking of $\mathcal{N}|| \mathrm{m} \cap \theta \mid \geq 1$, for each trap $\theta$ of $\mathcal{N}\}$

B. $\Omega(\mathcal{N}) \stackrel{\text { def }}{=}\{\mathrm{m}$ marking of $\mathcal{N}|| \mathrm{m} \cap \theta \mid=1$, for each mutex $\theta$ of $\mathcal{N}\}$.

Note that, since $\mathcal{N}$ is boolean, each marking can be represented as a set of places. Moreover, it is easy to check that $\Theta(\mathcal{N})$ and $\Omega(\mathcal{N})$ contain the initial marking $\mathrm{m}_{0}$ and are closed under the transition relation of the net. Thus both sets are inductive invariants of $\mathcal{N}$, that can be used to prove a safety property, by checking the emptiness of the intersection of the above sets with a set $\mathcal{E}$ of error markings.

Our method encodes the families of sets $\left\{\Theta(\mathcal{N}) \mid \mathcal{N} \in \llbracket \mathrm{b} \rrbracket_{\mathcal{R}}\right\}$ and $\{\Omega(\mathcal{N}) \mid$ $\left.\mathcal{N} \in \llbracket \mathrm{b} \rrbracket_{\mathcal{R}}\right\}$ by formulæ of WS $\kappa \mathrm{S}$, for a suitable integer constant $\kappa \geq 1$. To prove a parametric safety property given by a WS $\kappa S$ encoding of the $\mathcal{E}$ set, a sufficient (but not necessary) condition is that the WS $\kappa S$ formula defining the family of sets $\left\{\Theta(\mathcal{N}) \cap \Omega(\mathcal{N}) \cap \mathcal{E} \mid \mathcal{N} \in \llbracket \mathrm{b} \rrbracket_{\mathcal{R}}\right\}$ is unsatisfiable. Since automatatheoretic decision procedures exist for $\mathrm{WS} \kappa \mathrm{S}$ [19], we rely on existing provers 11] to perform this check.

Rewriting trees The crux of the method is to represent each predicateless behavioral term $\mathrm{t} \in[\mathrm{b}]_{\mathcal{R}}$ by a tree labeled with the rewriting rules from some rewriting sequence $\mathrm{b} \Leftarrow_{\mathcal{R}}^{*} \mathrm{t}$. As will be shown below, each such rewriting tree 


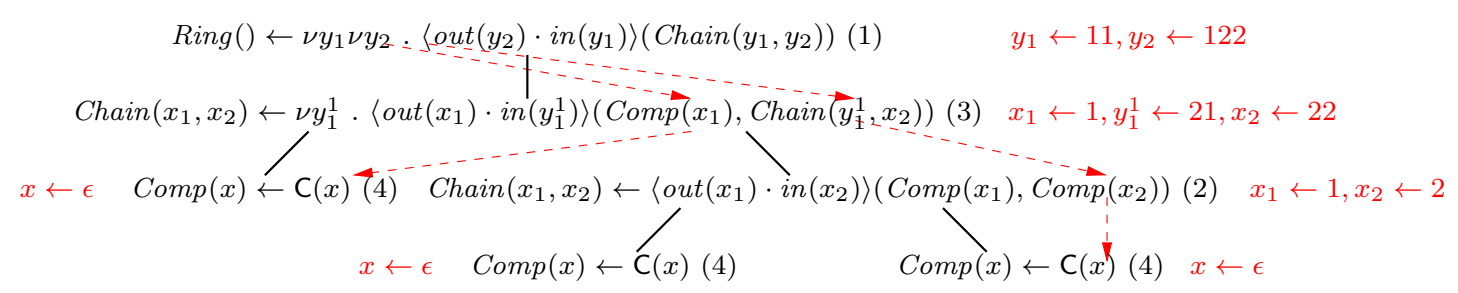

Fig. 3. A Rewriting Tree for the Token-Ring System

(Def. 3) defines an injective valuation $\nu: \mathbb{V}_{1} \rightarrow[1, \kappa]^{*}$ of the bound variables from $\mathrm{t}$ (Def. 4 that, in turn, induces a behavior $\mathrm{B}_{\nu}^{\mathrm{t}} \in \llbracket \mathrm{b} \rrbracket_{\mathcal{R}}$ (7). Since each term $\mathrm{t} \in[\mathrm{b}]_{\mathcal{R}}$ can be represented by a rewriting tree (Prop. 1), it follows that each behavior $\mathcal{N} \in \llbracket \mathrm{b} \rrbracket_{\mathcal{R}}$ corresponds to a rewriting tree (up to a permutation of component identifiers). Our encoding uses rewriting trees $\mathcal{T}$ as backbone parameters for the definition of the $\operatorname{trap}(\Theta(\mathcal{N}(\mathcal{T})))$ and mutex $(\Omega(\mathcal{N}(\mathcal{T})))$ invariant, respectively, where $\mathcal{N}(\mathcal{T})=\mathrm{B}_{\nu}^{\mathrm{t}}$ is the behavior induced by $\mathcal{T}$. In fact, any injective valuation of the variables is sufficient for safety checking, provided that the safety properties considered are defined by sets of error markings that are closed under permutations of component identifiers.

To simplify technicalities, we assume the existence of a rule $\mathrm{A}_{\mathrm{b}}\left(x_{1}, \ldots, x_{n}\right) \leftarrow$ $\mathrm{b}$ in $\mathcal{R}$, where $\mathrm{fv}(\mathrm{b})=\left\{x_{1}, \ldots, x_{n}\right\}$ and $\mathrm{A}_{\mathrm{b}}$ is a predicate symbol of arity $n$ not occurring elsewhere in $\mathcal{R}$. We also assume that the constant $\kappa$ is greater than the number of predicate atoms that occur in any rule of $\mathcal{R}$.

Definition 3. Given a rewriting system $\mathcal{R}$ and a behavioral term $\mathrm{b}$, a rewriting tree for $\mathrm{b}$ is a tree $\mathcal{T}:[1, \kappa]^{*} \rightarrow \mathcal{R}$, such that:

1. $\mathcal{T}(\epsilon)=\left(\mathrm{A}_{\mathrm{b}}\left(x_{1}, \ldots, x_{n}\right) \leftarrow \mathrm{b}\right)$

and, for all nodes $w \in \operatorname{nodes}(\mathcal{T})$, such that $\mathcal{T}(w)=\left(\mathrm{A}_{w}\left(x_{1}, \ldots, x_{\#\left(\mathrm{~A}_{w}\right)}\right) \leftarrow \mathrm{b}_{w}\right)$, the following hold:

2. for all $i \in\left[1, \#\right.$ pred $\left.\left(\mathrm{b}_{w}\right)\right]$, if $\operatorname{pred}_{i}\left(\mathrm{~b}_{w}\right)=\mathrm{A}_{w i}\left(y_{1}, \ldots, y_{\#\left(\mathrm{~A}_{w i}\right)}\right)$ then wi $\in$ $\operatorname{nodes}(\mathcal{T})$ and $\mathcal{T}(w i)=\left(\mathrm{A}_{w i}\left(x_{1}, \ldots, x_{\#\left(\mathrm{~A}_{w i}\right)}\right) \leftarrow \mathrm{b}_{w i}\right)$, for some rule of the form $\mathrm{A}_{w i}\left(x_{1}, \ldots, x_{\#\left(\mathrm{~A}_{w i}\right)}\right) \leftarrow \mathrm{b}_{w i}$ from $\mathcal{R}$.

3. for all $i \geq \#_{\text {pred }}\left(\mathrm{b}_{w}\right)$, we have wi $\notin \operatorname{nodes}(\mathcal{T})$.

We denote by $\mathbb{T}_{\mathcal{R}}(\mathrm{b})$ the set of rewriting trees for $\mathrm{b}$ in $\mathcal{R}$.

A rewriting tree $\mathcal{T}$ induces a characteristic term $\mathfrak{C}_{[\mathcal{T}]}$, obtained by the application of the rewriting rules labeling the tree nodes in some order of traversal, and a characteristic valuation $\nu_{[\mathcal{T}]}$, that maps each variable in the term to the node where it is instantiated.

Definition 4. Given a rewriting tree $\mathcal{T} \in \mathbb{T}_{\mathcal{R}}(\mathrm{b})$, the characteristic term $\mathfrak{C}_{[\mathcal{T}}$ and characteristic valuation $\nu_{[\mathcal{T}]}$ are defined inductively on the structure of $\mathcal{T}$ :

- if nodes $(\mathcal{T})=\{\epsilon\}$, then $\mathfrak{C}_{[\mathcal{T}]} \stackrel{\text { def }}{=} \mathcal{B}(x)$ and $\nu_{[\mathcal{T}]}(x) \stackrel{\text { def }}{=} \epsilon$, for $\mathcal{T}(\epsilon)=(\mathrm{A}(x) \leftarrow \mathcal{B}(x))$,

- else, let $1, \ldots, m$ be the children of the root of $\mathcal{T}$ and let:

$$
\begin{aligned}
\mathfrak{C}_{[\mathcal{T}]} \stackrel{\text { def }}{=} \nu y_{1} \ldots \nu y_{n} \cdot\langle\Gamma\rangle\left(\mathfrak{C}_{\left[\mathcal{T}_{1}\right]}\left[z_{1}^{1} / x_{1}, \ldots, z_{\#\left(\mathrm{~A}_{1}\right)}^{1} / x_{\#\left(\mathrm{~A}_{1}\right)}\right], \ldots,\right. \\
\left.\mathfrak{C}_{\left[\mathcal{I}_{m}\right]}\left[z_{1}^{m} / x_{1}, \ldots, z_{\#\left(\mathrm{~A}_{1}\right)}^{m} / x_{\#\left(\mathrm{~A}_{m}\right)}\right]\right)
\end{aligned}
$$




$$
\begin{gathered}
\text { where } \nu_{[\mathcal{T}]}\left(z_{j}^{i}\right) \stackrel{\text { def }}{=} i \cdot \nu_{\left[\mathcal{T}_{i}\right]}\left(x_{j}\right), \text { for all } i \in[1, m] \text { and } j \in\left[1, \#\left(\mathrm{~A}_{i}\right)\right] \text {, such that } \\
\qquad \begin{array}{c}
\mathcal{T}(\epsilon)=\left(\mathrm { A } ( x _ { 1 } , \ldots , x _ { \# ( \mathrm { A } ) } ) \leftarrow \nu y _ { 1 } \ldots \nu y _ { n } \cdot \langle \Gamma \rangle \left(\mathrm{A}_{1}\left(z_{1}^{1}, \ldots, z_{\#\left(\mathrm{~A}_{1}\right)}^{1}\right), \ldots,\right.\right. \\
\left.\mathrm{A}_{m}\left(z_{1}^{m}, \ldots, z_{\#\left(\mathrm{~A}_{m}\right)}^{m}\right)\right)
\end{array}
\end{gathered}
$$

A consequence of the specific form of rewriting rules (Def. 1) is that each (free or bound) variable $y$ from $\mathfrak{C}_{[\mathcal{T}]}$ is mapped by $\nu_{[\mathcal{T}]}$ to a unique node $w \in \operatorname{nodes}\left(\mathfrak{C}_{[\mathcal{T}]}\right)$, such that $\mathfrak{C}_{[\mathcal{T}]}(w)$ contains an instance atom $\mathcal{B}(x)$, where $x$ is substituted with $y$ along the path from the node $u$ that introduces $y$ (take $u=\epsilon$ if $y \in \operatorname{fv}\left(\mathfrak{C}_{[\mathcal{T}]}\right)$ ) to $w$. For example, Fig. 3 shows the rewriting tree for the rewriting sequence from Example 3; we annotate on the side of the tree the bottom-up definition of the characteristic valuation associating the bound variables $y_{1}$ and $y_{2}$ with the nodes of the rewriting tree where they are instantiated.

Below we show that the set $[\mathrm{b}]_{\mathcal{R}}$ of predicateless terms obtained by complete rewriting is the same as the set of characteristic terms that correspond to some rewriting tree:

Proposition 1. Given a behavioral term $\mathrm{b}$, we have $[\mathrm{b}]_{\mathcal{R}}=\left\{\mathfrak{C}_{[\mathcal{T}]} \mid \mathcal{T} \in \mathbb{T}_{\mathcal{R}}(\mathrm{b})\right\}$.

Its proof is an easy consequence of the confluence of the rewriting system, i.e. the order in which the rules are applied to a term does not change the resulting predicateless term.

\subsection{Encoding Invariants and Error Configurations}

We begin by building a WS $\kappa S$ formula that describes an infinite $\kappa$-ary tree whose finite prefix encodes a rewriting tree $\mathcal{T} \in \mathbb{T}_{\mathcal{R}}$ (b). Let us assume that $\mathcal{R}=$ $\left\{\mathrm{r}_{1}, \ldots, \mathrm{r}_{N}\right\}$, such that $\mathrm{r}_{1}=\left(\mathrm{A}_{\mathrm{b}}\left(x_{1}, \ldots, x_{n}\right) \leftarrow \mathrm{b}\right)$. We use a designated tuple of second order variables $\mathbf{U}=\left\langle U_{1}, \ldots, U_{N}\right\rangle$, where each variable $U_{i}$ is interpreted as the set of tree nodes labeled with the rule $r_{i}$ in $\mathcal{T}$. With this convention, the $R \operatorname{Tree}(\mathbf{U})$ formula (Fig. 4 ) defines a rewriting tree:

- line (8) states that the sets $\mathbf{U}$ are pairwise disjoint and that $U_{1}$ is a singleton containing the root of the tree (condition 1 of Def. 3 ).

- line (9) states that the union of the sets $\mathbf{U}$ is prefix-closed, i.e. the parent $x$ of each node $x . \ell$ from some $U_{i}$ belongs to some $U_{j}$, for $i, j \in[1, N]$.

- lines (10) and (11) encode the conditions 2 and 3 of Def. 3 , respectively.

Clearly, for each model $\nu$ of $R$ Tree $(\mathbf{U})$, there is a unique rewriting tree, denoted $\mathcal{T}_{\nu}^{\mathrm{U}} \in \mathbb{T}_{\mathcal{R}}(\mathrm{b})$, such that $\operatorname{nodes}\left(\mathcal{T}_{\nu}^{\mathrm{U}}\right)=\bigcup_{i=1}^{N} \nu\left(U_{i}\right)$ and $\mathcal{T}_{\nu}^{\mathrm{U}}(w)=\mathrm{r}_{i}$ iff $w \in \nu\left(U_{i}\right)$, for all $i \in[1, N]$ and $w \in \operatorname{nodes}\left(\mathcal{T}_{\nu}^{\mathrm{U}}\right)$.

As said, a rewriting tree $\mathcal{T} \in \mathbb{T}_{\mathcal{R}}$ (b) defines a behavior (i.e. a marked PN) denoted by $\mathcal{N}(\mathcal{T}) \stackrel{\text { def }}{=} \mathrm{B}_{\nu}^{\mathrm{t}}(6)$, where $\mathrm{t}=\mathfrak{C}_{[\mathcal{T}]}$ is the characteristic term and $\nu=\nu_{[\mathcal{T}]}$ is the characteristic valuation of $\mathcal{T}$ (Def. 4). An invariant of $\mathcal{N}(\mathcal{T})$ is a set of markings, i.e. a set of sets of marked places from different components.

Let $\left\{\mathcal{B}_{i} \stackrel{\text { def }}{=}\left\langle\mathcal{P}_{i}, \mathcal{S}_{i}, \mathcal{I}_{i}, \Delta_{i}\right\rangle\right\}_{i=1}^{K}$ be the set of component types that occur in the rules of $\mathcal{R}$ and let $\mathbf{Z}=\left\langle Z_{1}, \ldots, Z_{K}\right\rangle$ be a tuple of second-order variables, where $Z_{i}$ is interpreted as the set of identifiers of the components of type $\mathcal{B}_{i}$. 


$$
\begin{aligned}
& \operatorname{RTree}(\mathbf{U}) \stackrel{\text { def }}{=} \forall x . \bigwedge_{1 \leq i<j \leq N}\left(\neg U_{i}(x) \vee \neg U_{j}(x)\right) \wedge U_{1}(x) \leftrightarrow x=\epsilon \wedge \\
& \forall x \cdot \bigwedge_{i: \mathrm{r}_{i} \in \mathcal{R}} \bigwedge_{\ell=1}^{\kappa} U_{i}(x . \ell) \rightarrow \bigvee_{\mathrm{r}_{j} \in \mathcal{R}} U_{j}(x) \wedge
\end{aligned}
$$

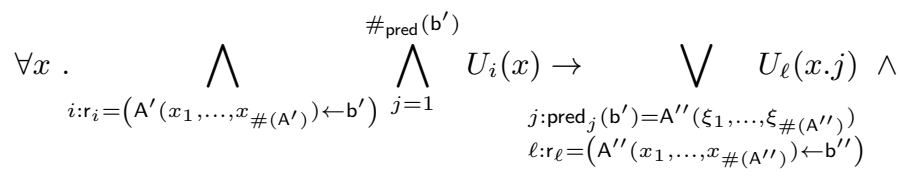

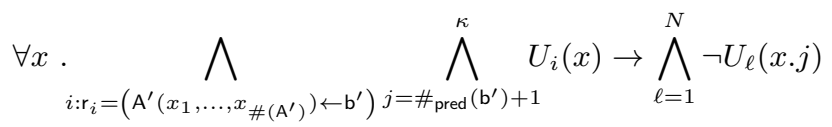

Fig. 4. The Definition of Rewriting Trees

We encode the markings of $\mathcal{N}(\mathcal{T})$ by a WS $\kappa$ S formula using a tuple of secondorder variables $\mathbf{X}=\left\langle X_{S} \mid S \in \bigcup_{i=1}^{K} \mathcal{S}_{i}\right\rangle$, where each $X_{S}$ is interpreted as the set of identifiers of the components currently in state $S(w)$ and define the set $\sigma_{\nu}^{\mathbf{x}} \stackrel{\text { def }}{=}\left\{S(w) \mid S \in \bigcup_{i=1}^{K} \mathcal{S}_{i}, w \in \nu\left(X_{S}\right)\right\}$. The following formula constrains the set represented by $\mathbf{X}$ to be a marking of $\mathcal{N}\left(\mathcal{T}_{\nu}^{\mathrm{U}}\right)$ :

$$
\begin{aligned}
\operatorname{mark}(\mathbf{X}, \mathbf{Z}) & \stackrel{\text { def }}{=} \forall x . \bigwedge_{S \neq S^{\prime} \in \cup_{j=1}^{K} \mathcal{S}_{j}}\left(\neg X_{S}(x) \vee \neg X_{S^{\prime}}(x)\right) \wedge \bigvee_{S \in \cup_{j=1}^{K} \mathcal{S}_{j}} X_{S}(x) \leftrightarrow \bigvee_{j=1}^{K} Z_{j}(x) \\
\operatorname{inst}(\mathbf{Z}, \mathbf{U}) & \stackrel{\text { def }}{=} \forall x . \bigwedge_{i=1}^{K} Z_{i}(x) \leftrightarrow \bigvee_{j: r_{j}=\left(\mathrm{A}^{\prime}(y) \leftarrow \mathcal{B}_{i}(y)\right)} U_{j}(x)
\end{aligned}
$$

Intuitively, $\operatorname{mark}(\mathbf{X}, \mathbf{Z})$ states that no component can be in two different states (first conjunct) and each component is an instance of some component type (second conjunct). The formula $\operatorname{inst}(\mathbf{Z}, \mathbf{U})$ above relates the instance indices to the nodes of the rewriting tree where the corresponding instance atoms occur, assuming that the sets $\mathbf{U}$ are constrained by RTree $(\mathbf{U})$. Then, for each model $\nu$ of $\operatorname{mark}(\mathbf{X}, \mathbf{Z}) \wedge \operatorname{inst}(\mathbf{Z}, \mathbf{U}) \wedge R \operatorname{Rree}(\mathbf{U})$, the set $\sigma_{\nu}^{\mathbf{x}}$ is a marking of $\mathcal{N}\left(\mathcal{T}_{\nu}^{\mathbf{U}}\right)$.

We proceed with the encoding of invariants and error states, by assuming the existence of a flow formula, that defines the pre- and post-sets of the transitions from a behavior $\mathcal{N}\left(\mathcal{T}_{\nu}^{\mathrm{U}}\right)$, formally described next $(4.2)$. In the following, the primed copy of the tuple $\mathbf{X}$ is denoted as $\mathbf{X}^{\prime}$.

Definition 5. $\Phi\left(\mathbf{X}, \mathbf{X}^{\prime}, \mathbf{U}\right)$ is a flow formula for $\mathrm{b}$ and $\mathcal{R}$ if, for each model $\nu$ of RTree $(\mathbf{U})$, we have $\nu \mid=\Phi\left(\mathbf{X}, \mathbf{X}^{\prime}, \mathbf{U}\right)$ if and only if ${ }^{\bullet} t=\sigma_{\nu}^{\mathbf{X}}$ and $t^{\bullet}=\sigma_{\nu}^{\mathbf{X}^{\prime}}$, for some transition $t$ of $\mathcal{N}\left(\mathcal{T}_{\nu}^{\mathrm{U}}\right)$.

Given a flow formula $\Phi$, the parametric trap invariant $\operatorname{TrapInv}^{\Phi}(\mathbf{X}, \mathbf{U})$ is defined by the formula below:

$$
\begin{array}{r}
\operatorname{trap}^{\Phi}(\mathbf{X}, \mathbf{U}) \stackrel{\text { def }}{=} \forall \mathbf{Y}^{1}, \mathbf{Y}^{2} . \Phi\left(\mathbf{Y}^{1}, \mathbf{Y}^{2}, \mathbf{U}\right) \wedge \operatorname{inter}\left(\mathbf{X}, \mathbf{Y}^{1}\right) \rightarrow \operatorname{inter}\left(\mathbf{X}, \mathbf{Y}^{2}\right) \\
\operatorname{TrapInv}^{\Phi}(\mathbf{X}, \mathbf{U}) \stackrel{\text { def }}{=} \exists \mathbf{Z} . \operatorname{mark}(\mathbf{X}, \mathbf{Z}) \wedge \forall \mathbf{Y}^{1}, \mathbf{Y}^{2} \cdot \operatorname{init}\left(\mathbf{Y}^{1}, \mathbf{Z}\right) \wedge \operatorname{inter}\left(\mathbf{Y}^{1}, \mathbf{Y}^{2}\right) \wedge \\
\quad \operatorname{trap}^{\Phi}\left(\mathbf{Y}^{2}, \mathbf{U}\right) \rightarrow \operatorname{inter}\left(\mathbf{X}, \mathbf{Y}^{2}\right)
\end{array}
$$


where the tuples $\mathbf{Y}^{i} \stackrel{\text { def }}{=}\left\langle Y_{S}^{i} \mid S \in \bigcup_{j=1}^{K} \mathcal{S}_{j}\right\rangle$, for $i=1,2$, are distinct copies of $\mathbf{X}$. The auxiliary formula $\operatorname{init}(\mathbf{X}, \mathbf{Z}) \stackrel{\text { def }}{=} \operatorname{mark}(\mathbf{X}, \mathbf{Z}) \wedge \bigwedge_{j=1}^{K} \forall x . Z_{j}(x) \leftrightarrow X_{\mathcal{I}_{j}}(x)$ states that $\mathbf{X}$ represents the initial marking of the behavior, whereas inter $(\mathbf{X}, \mathbf{Y}) \stackrel{\text { def }}{=}$ $\exists x . \bigvee_{j=1}^{K} \bigvee_{S \in \mathcal{S}_{j}} X_{S}(x) \wedge Y_{S}(x)$ means that the sets of places encoded by $\mathbf{X}$ and $\mathbf{Y}$, respectively, have a non-empty intersection. Intuitively, the formula $\operatorname{trap}^{\Phi}(\mathbf{X}, \mathbf{U})$ defines the traps (point 1 of Def. 2), whereas $\operatorname{TrapInv}^{\Phi}(\mathbf{X}, \mathbf{U})$ defines the set of markings that intersect with the initial marking and with each trap of the behavior, i.e. the trap invariant (point A of Def. 2

Mutexes and mutex invariants (points 2 and $B$ of Def. 2 ) are defined by the formulæ:

$$
\begin{array}{r}
\left.\operatorname{mutex}^{\Phi}(\mathbf{X}, \mathbf{U}) \stackrel{\text { def }}{=} \forall \mathbf{Y}^{1}, \mathbf{Y}^{2} . \Phi\left(\mathbf{Y}^{1}, \mathbf{Y}^{2}, \mathbf{U}\right) \rightarrow \wedge \begin{array}{c}
\neg \operatorname{inter}\left(\mathbf{X}, \mathbf{Y}^{1}\right) \leftrightarrow \\
\operatorname{single}\left(\mathbf{X}, \mathbf{Y}^{1}\right) \leftrightarrow \operatorname{sinter}\left(\mathbf{X}, \mathbf{Y}^{2}\right)
\end{array}\right) \\
\operatorname{MutexInv}^{\Phi}(\mathbf{X}, \mathbf{U}) \stackrel{\text { def }}{=} \exists \mathbf{Z} . \operatorname{mark}(\mathbf{X}, \mathbf{Z}) \wedge \forall \mathbf{Y}^{1}, \mathbf{Y}^{2} . \operatorname{init}\left(\mathbf{Y}^{1}, \mathbf{Z}\right) \wedge \operatorname{single}\left(\mathbf{Y}^{1}, \mathbf{Y}^{2}\right) \wedge \\
\operatorname{mutex}^{\Phi}\left(\mathbf{Y}^{2}, \mathbf{U}\right) \rightarrow \operatorname{single}\left(\mathbf{X}, \mathbf{Y}^{2}\right)
\end{array}
$$

where single $(\mathbf{X}, \mathbf{Y}) \stackrel{\text { def }}{=} \exists_{1} x . \bigvee_{j=1}^{K} \bigvee_{S \in \mathcal{S}_{j}} X_{S}(x) \wedge Y_{S}(x)$ states that the intersection of the sets of places defined by $\mathbf{X}$ and $\mathbf{Y}$ is a singletor ${ }^{3}$ The following lemma states the correctness of the encoding:

Lemma 1. Given a flow formula $\Phi\left(\mathbf{X}, \mathbf{X}^{\prime}, \mathbf{U}\right)$ and a model $\nu$ of $\operatorname{RTree}(\mathbf{U}) \wedge$ inst $(\mathbf{Z}, \mathbf{U})$, we have:

1. $\Theta\left(\mathcal{N}\left(\mathcal{T}_{\nu}^{\mathbf{U}}\right)\right)=\left\{\sigma_{\mu}^{\mathbf{X}} \mid \mu \models \operatorname{Trap} \operatorname{Inv}^{\Phi}(\mathbf{X}, \mathbf{U}), \mu(\mathbf{U} \cdot \mathbf{Z})=\nu(\mathbf{U} \cdot \mathbf{Z})\right\}$,

2. $\Omega\left(\mathcal{N}\left(\mathcal{T}_{\nu}^{\mathbf{U}}\right)\right)=\left\{\sigma_{\mu}^{\mathbf{X}} \mid \mu=\operatorname{MutexInv}^{\Phi}(\mathbf{X}, \mathbf{U}), \mu(\mathbf{U} \cdot \mathbf{Z})=\nu(\mathbf{U} \cdot \mathbf{Z})\right\}$.

In our examples $(\$ 5$ we consider two kinds of error sets, defined as:

$$
\begin{aligned}
& \operatorname{DeadLock}^{\Phi}(\mathbf{X}, \mathbf{U}) \stackrel{\text { def }}{=} \forall \mathbf{Y}^{1}, \mathbf{Y}^{2} . \Phi\left(\mathbf{Y}^{1}, \mathbf{Y}^{2}, \mathbf{U}\right) \rightarrow \exists x \cdot \bigvee_{j=1}^{K} \bigvee_{S \in \mathcal{S}_{j}} Y_{S}^{1}(x) \wedge \neg X_{S}(x) \\
& \text { CriticalSection }^{\Xi}(\mathbf{X}, \mathbf{U}) \stackrel{\text { def }}{=} \exists x \exists y . \bigvee_{S, S^{\prime} \in \Xi} X_{S}(x) \wedge X_{S^{\prime}}(y) \wedge \neg x=y
\end{aligned}
$$

Intuitively, DeadLock ${ }^{\Phi}(\mathbf{X}, \mathbf{U})$ defined the deadlock markings, in which no transition of the behavior is enabled and CriticalSection ${ }^{\Xi}(\mathbf{X}, \mathbf{U})$ states that no two components are at the same time in a state from a given set $\Xi \subseteq \bigcup_{i=1}^{K} \mathcal{S}_{i}$ of state types. It is worth mentioning that these sets of error markings are closed under permutations of component indices 4 , which makes them suitable for safety checking using our encoding of trap and mutex invariants (with variables mapped to the nodes of the rewriting tree where they occur instantiated, as in Fig. 3.

\subsection{The Flow of a Behavioral Term}

The previous definition of structural invariants relied on the existence of a flow formula $\Phi\left(\mathbf{X}, \mathbf{X}^{\prime}, \mathbf{U}\right)$, stating that $\sigma_{\nu}^{\mathbf{X}}$ and $\sigma_{\nu}^{\mathbf{X}^{\prime}}$ are the pre- and post-sets of some transition from the behavior (i.e. marked PN) $\mathcal{N}\left(\mathcal{T}_{\nu}^{\mathrm{U}}\right)$, whenever $\nu$ is a model of $\operatorname{RTree}(\mathbf{U}) \wedge \Phi\left(\mathbf{X}, \mathbf{X}^{\prime}, \mathbf{U}\right)$ (Def. 5). In this section, we describe the flow formula. In the following, we assume w.l.o.g. that the inductive rules in $\mathcal{R}$ are of the form:

$$
\mathrm{A}\left(x_{1}, \ldots, x_{\#(\mathrm{~A})}\right) \leftarrow \nu y_{1} \ldots \nu y_{m} \cdot\left\langle\sum_{k=1}^{m} P_{k 1}\left(x_{k 1}\right) \cdot \ldots \cdot P_{k r_{k}}\left(x_{k r_{k}}\right)\right\rangle\left(\mathrm{t}_{1}, \ldots, \mathrm{t}_{n}\right)
$$

$\overline{3} \exists_{1} x . \phi(x)$ is a shorthand for $\exists x . \phi(x) \wedge \forall x \forall y . \phi(x) \wedge \phi(y) \rightarrow x=y$.

${ }^{4}$ This is the case for every WS $\kappa S$ formula consisting of equality and membership atoms, without successor functions. 


$$
\begin{gathered}
\Phi\left(\mathbf{X}, \mathbf{X}^{\prime}, \mathbf{U}\right) \stackrel{\text { def }}{=} \bigvee_{\ell=1}^{N} \bigvee_{\pi \in \operatorname{lnter}\left(\mathrm{r}_{\ell}\right)} \Psi_{\ell, \pi}\left(\mathbf{X}, \mathbf{X}^{\prime}, \mathbf{U}\right) \\
\Psi_{\ell,\left\{P_{1}\left(x_{1}\right), \ldots, P_{n}\left(x_{n}\right)\right\}}\left(\mathbf{X}, \mathbf{X}^{\prime}, \mathbf{U}\right) \stackrel{\text { def }}{=} \exists y_{0} \ldots \exists y_{n} \cdot U_{\ell}\left(y_{0}\right) \wedge \\
\bigwedge_{i=1}^{n}\left(\bigvee_{\mathrm{r}^{\prime}=\left(\mathrm{A}^{\prime}\left(y_{i}\right) \leftarrow \mathcal{B}\left(y_{i}\right)\right)} \operatorname{Path}_{\mathrm{r}_{\ell}, \mathrm{r}^{\prime}}^{x_{i}, y_{i}}\left(y_{0}, y_{i}, \mathbf{U}\right)\right) \wedge \\
\forall x . \bigwedge_{S \in \bigcup_{j=1}^{K}}\left[( X _ { S } ( x ) \leftrightarrow \mathcal { S } _ { P _ { j } = S } x = y _ { k } ) \wedge \left(X_{S}^{\prime}(x) \leftrightarrow \bigvee_{P_{k}}^{\bullet} x=S\right.\right.
\end{gathered}
$$

Fig. 5. Definition of the Flow Formula for the Rewriting System $\mathcal{R}=\left\{\mathrm{r}_{1}, \ldots, \mathrm{r}_{N}\right\}$.

if necessary, by applying the flattening relation $(5)$ to each rule of $\mathcal{R}$. We denote by $\operatorname{Inter}(\mathrm{r}) \stackrel{\text { def }}{=}\left\{\left\{P_{k i}\left(x_{k i}\right) \mid i \in\left[1, r_{k}\right]\right\} \mid k \in[1, m]\right\}$ the set of sets of port atoms $P_{k i}\left(x_{k i}\right)$, corresponding to the interactions (i.e. the monomials from the architecture) from $r$.

The flow formula $\Phi\left(\mathbf{X}, \mathbf{X}^{\prime}, \mathbf{U}\right)$ is given in Fig. 5 Essentially, the formula 12 is split into a disjunction of formulæ $\Psi_{\ell,\left\{P_{1}\left(x_{1}\right), \ldots, P_{n}\left(x_{n}\right)\right\}}(13)$, one for each rule $\boldsymbol{r}_{\ell} \in \mathcal{R}$ and each set of port atoms $\left\{P_{1}\left(x_{1}\right), \ldots, P_{n}\left(x_{n}\right)\right\}$, denoting an interaction (monomial) from $r_{\ell}$. To understand the formulæ (13), recall that each of the variables $x_{1}, \ldots, x_{n}$ is mapped to the (unique) node of the rewriting tree containing an instance atom $\mathcal{B}_{i}\left(x_{i}\right)$. In order to find this node, we must track the variable $x_{i}$ from the node labeled by the rule $\mathrm{r}$, to the node where this instance atom occurs. This is done by the $\operatorname{Path}_{\mathrm{r}_{\ell}, \mathrm{r}^{\prime}}^{x_{i}, y_{i}}\left(y_{0}, y_{i}, \mathbf{U}\right)$ formulæ 14, that holds whenever $\mathcal{T}_{\nu}^{\mathrm{U}}$ is a rewriting tree, uniquely encoded by the interpretation $\nu$ of the $\mathbf{U}$ variables, and $y_{0}, y_{i}$ are mapped to the source and the destination of a path from a node $w \in \operatorname{nodes}\left(\mathcal{T}_{\nu}^{\mathrm{U}}\right)$, with label $\mathcal{T}_{\nu}^{\mathrm{U}}(w)=\mathrm{r}_{\ell}$ to a node $w^{\prime} \in \operatorname{nodes}\left(\mathcal{T}_{\nu}^{\mathrm{U}}\right)$, with label $\mathcal{T}_{\nu}^{\mathrm{U}}\left(w^{\prime}\right)=\mathrm{r}^{\prime}$, such that $x_{i}$ and $y_{i}$ are variables that occur in the bodies of $\mathrm{r}$ and $\mathrm{r}^{\prime}$, respectively, mapped to the same variable in the characteristic term of $\mathcal{T}_{\nu}^{\mathrm{U}}$ (Def. 4). We describe these paths by an automaton and define $\operatorname{Path}_{\mathrm{r}_{\ell}, \mathrm{r}^{\prime}}^{x_{i}, y_{i}}\left(y_{0}, y_{i}, \mathbf{U}\right)$, by translating this automaton into a WS $\kappa S$ formula.

But first, let us define paths in a tree formally. Given a $\kappa$-ary tree $\mathcal{T}$, a path is a finite sequence of nodes $\rho=n_{1}, \ldots, n_{\ell} \in \operatorname{nodes}(\mathcal{T})$, such that, for all $i \in[1, \ell-1], n_{i+1}$ is either the parent $\left(n_{i}=n_{i+1} \alpha_{i}\right)$ or a child $\left(n_{i+1}=n_{i} \alpha_{i}\right)$ of $n_{i}$, for some $\alpha_{i} \in[1, \kappa]$. The path is determined by the source node and the sequence $\left(\alpha_{1}, d_{1}\right) \ldots\left(\alpha_{\ell-1}, d_{\ell-1}\right)$ of directions $\left(\alpha_{i}, d_{i}\right) \in[1, \kappa] \times\{\uparrow, \downarrow\}$, with the following meaning: $d_{i}=\uparrow$ if $n_{i+1} \alpha_{i}=n_{i}$ and $d_{i}=\downarrow$ if $n_{i+1}=n_{i} \alpha_{i}$. Given two distinct nodes $w_{1}, w_{2} \in \operatorname{nodes}(\mathcal{T})$, there is a unique minimal path from $w_{1}$ to $w_{2}$, labeled by a sequence denoted as $\rho\left(w_{1}, w_{2}\right)$. This path climbs from $w_{1}$ to the greatest common prefix $w$ of $w_{1}$ and $w_{2}$, before descending from $w$ to $w_{2}$.

A path automaton is a tuple $A=(Q, I, F, \delta)$, where $Q$ is a set of states, $I, F \subseteq Q$ are the sets of initial and final states, respectively, and $\delta \subseteq Q \times[1, \kappa] \times\{\uparrow, \downarrow\} \times Q$ is a set of transitions $q \stackrel{(\alpha, d)}{\longrightarrow} q^{\prime}$, with $\alpha \in[1, \kappa]$ being a direction and $d \in\{\uparrow, \downarrow\}$ indicating whether the automaton moves up or down in the tree. A run of $A$ over $\omega=\left(\alpha_{1}, d_{1}\right) \ldots\left(\alpha_{n-1}, d_{n-1}\right)$ is a sequence of states $q_{1}, \ldots, q_{n} \in Q$ such that $q_{1} \in I$ and $q_{i} \stackrel{\left(\alpha_{i}, d_{i}\right)}{\longrightarrow} q_{i+1} \in \delta$, for all $i \in[1, n-1]$. The run is accepting if and only if $q_{n} \in F$. The language $\mathcal{L}(A)$ of $A$ is the set of sequences over which $A$ has an accepting run. 
A path automaton $A=(Q, I, F, \delta)$ corresponds (Lemma 2$)$ to the formula in Figure 6. that can be effectively built from the description of $A$. Here $Q=\left\{q_{1}, \ldots, q_{L}\right\}$ is the

$$
\begin{aligned}
& \Delta_{A}(x, y, \mathbf{Y}) \stackrel{\text { def }}{=} \bigwedge_{1 \leq i \neq j \leq L} \forall z \cdot\left(\neg Y_{i}(z) \vee \neg Y_{j}(z) \wedge \bigvee_{q_{i} \in I} Y_{i}(x) \wedge \bigvee_{q_{j} \in F} Y_{j}(y) \wedge\right. \\
& \bigwedge_{i=1}^{L} \forall z \cdot z \neq y \wedge Y_{i}(z) \rightarrow \\
& \bigwedge_{j=1}^{L} \forall z \cdot z \neq x \wedge Y_{j}(z \cdot \alpha) \vee Y_{j}(z) \rightarrow q_{j: q_{i} \stackrel{(\alpha, \uparrow)}{\longrightarrow} q_{j}} \exists z^{\prime} \cdot z^{\prime} \cdot \alpha=z \wedge Y_{j}\left(z^{\prime}\right) \wedge \\
& \bigvee_{j} \exists z^{\prime} \cdot z^{\prime} . \alpha=z \wedge Y_{i}\left(z^{\prime}\right) \vee \bigvee_{i: q_{i} \stackrel{(\alpha, \downarrow)}{\longrightarrow} q_{j}} \bigvee_{i, \uparrow)} Y_{i}(z . \alpha)
\end{aligned}
$$

Fig. 6. Definition of the Path Automaton formula $\Delta_{A}(x, y, \mathbf{Y})$

set of states of $A$ and $\mathbf{Y}=\left\langle Y_{1}, \ldots, Y_{L}\right\rangle$ are second order variables interpreted as the sets of tree nodes labeled by the automaton with $q_{1}, \ldots, q_{L}$, respectively. Intuitively, the first three conjuncts of the above formula 16 encode the facts that $\mathbf{Y}$ are disjoint (no tree node is labeled by more than one state during the run) and that the run starts in an initial state with node $x$ and ends in a final state with node $y$. The fourth conjunct (17) states that, for every non-final node on the path, if the automaton visits that node by state $q_{i}$, then either the node has a $(\alpha, \downarrow)$-child or a $(\alpha, \uparrow)$-parent visited by state $q_{j}$, where $q_{i} \stackrel{(\alpha, \downarrow)}{\longrightarrow} q_{j}$ and $q_{i} \stackrel{(\alpha, \uparrow)}{\longrightarrow} q_{j}$ are transitions of the automaton. The fifth conjunct $(18)$ is the reversed flow condition on the path, needed to ensure that the sets $\mathbf{Y}$ do not contain useless nodes, being thus symmetric to the fourth. The following result stems from the classical automata-logic connection ${ }^{5}[14, \S 2.10]$ :

Lemma 2. Given a tree $\mathcal{T}$ with $\operatorname{nodes}(\mathcal{T}) \subseteq[1, \kappa]^{*}$ and a sequence $\omega \in([1, \kappa] \times\{\uparrow, \downarrow\})^{*}$ from $w_{1} \in \operatorname{nodes}(\mathcal{T})$ to $w_{2} \in \operatorname{nodes}(\mathcal{T})$, for each valuation $\nu$ such that $\nu(x)=w_{1}$ and $\nu(y)=w_{2}$, we have $\omega \in \mathcal{L}(A) \Longleftrightarrow \nu \mid=\exists \mathbf{Y} . \Delta_{A}(x, y, \mathbf{Y})$.

Our purpose is to define path automata that recognize the paths between the node where a bound variable is introduced and the node where the variable is instantiated, in a given rewriting tree. This automaton is directly inferred from the syntax of the rules in $\mathcal{R}$. For each pair of rules $r_{1}, r_{2} \in \mathcal{R}$ and variables $z_{1}, z_{2} \in \mathbb{V}_{1}$ that occur in the bodies of $\mathrm{r}_{1}$ and $\mathrm{r}_{2}$, respectively, we define the path automaton $A_{\mathrm{r}_{1}, \mathrm{r}_{2}}^{z_{1}, z_{2}} \stackrel{\text { def }}{=}\left(Q, I_{\mathrm{r}_{1}}^{z_{1}}, F_{\mathrm{r}_{2}}^{z_{2}}, \delta\right)$ :

- We associate a state $q_{\mathrm{r}, z}^{d}$ to each rule $\mathrm{r}=\left(\mathrm{A}\left(x_{1}, \ldots, x_{\# \mathrm{~A}}\right) \leftarrow \overline{\mathrm{b}}\right)$, each variable $z$ occurring (free or bound) in $\overline{\mathrm{b}}$ and each direction $d \in\{\uparrow, \downarrow\}$. The intuition is that the automaton visits the state $q_{r, z}^{d}$ while going up or down, as indicated by the direction $d$, currently tracking variable $z$ in rule $r$.

${ }^{5}$ A similar conversion of tree walking automata to MSO has been described in 12 . 

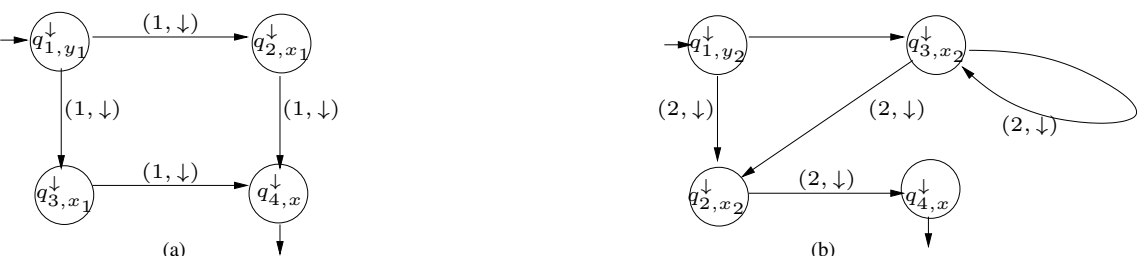

Fig. 7. Path Automata Recognizing the Instantiation Paths from Example 1

- The sets of initial and final states are $I_{\mathrm{r}_{1}}^{z_{1}} \stackrel{\text { def }}{=}\left\{q_{\mathrm{r}_{1}, z_{1}}^{d} \mid d=\uparrow, \downarrow\right\}$ and $F_{\mathrm{r}_{2}}^{z_{2}} \stackrel{\text { def }}{=}\left\{q_{\mathrm{r}_{2}, z_{2}}^{\downarrow}\right\}$. In other words, the automaton starts to track $z_{1}$ in $r_{1}$, moving either up or down and it ends tracking $z_{2}$ in $\mathrm{r}_{2}$, while moving down.

- The transitions are $q_{\mathrm{r}_{1}, y_{j}}^{\downarrow} \stackrel{(\alpha, \downarrow)}{\longrightarrow} q_{\mathrm{r}_{2}, x_{j}}^{\downarrow}, q_{\mathrm{r}_{2}, x_{j}}^{\uparrow} \stackrel{(\alpha, \uparrow)}{\longrightarrow} q_{\mathrm{r}_{1}, y_{j}}^{\uparrow}$ and $q_{\mathrm{r}_{2}, x_{j}}^{\uparrow} \stackrel{(\alpha, \uparrow)}{\longrightarrow} q_{\mathrm{r}_{1}, y_{j}}^{\downarrow}$, for any two distinct rules $\mathrm{r}_{i}=\left(\mathrm{A}_{j}\left(x_{1}, \ldots, x_{\#(\mathrm{~A})}\right) \leftarrow \mathrm{b}_{i}\right), i=1,2$, all $\alpha \in\left[1\right.$, \# pred $\left.\left(\mathrm{b}_{1}\right)\right]$, such that $\operatorname{pred}_{\alpha}\left(\mathrm{b}_{1}\right)=\mathrm{A}_{2}\left(y_{1}, \ldots, y_{\#\left(\mathrm{~A}_{2}\right)}\right)$ and all $j \in\left[1, \#\left(\mathrm{~A}_{2}\right)\right]$. Intuitively, if $\mathrm{r}_{1}$ labels the parent of the node labeled by $r_{2}$ in the rewriting tree, the automaton can move either: (i) down from tracking $y_{j}$ in $r_{1}$ to tracking $x_{j}$ in $r_{2}$, (ii) up from tracking $x_{j}$ in $r_{2}$ to tracking $y_{j}$ in $r_{1}$, or (iii) change direction from moving up tracking $x_{j}$ in $r_{2}$ to moving down tracking $y_{j}$ in $r_{1}$. In particular, the last case might be needed to accept a path that only goes up in the tree.

Note that a run of a path automaton $A_{\mathrm{r}_{1}, \mathrm{r}_{2}}^{z_{1}, z_{2}}$ may have at most one change of direction, by a rule of the form $q_{\mathrm{r}_{2}, x_{j}}^{\uparrow} \stackrel{(\alpha, \uparrow)}{\longrightarrow} q_{\mathrm{r}_{1}, y_{j}}^{\downarrow}$.

Example 4. The paths that track the instantiations of the variables $y_{1}$ and $y_{2}$ in a rewriting tree for the term $\operatorname{Ring}()$ are depicted in dashed lines in Fig. 3 . The path

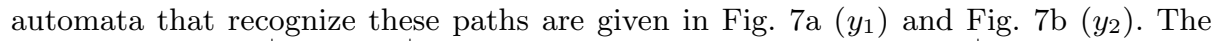

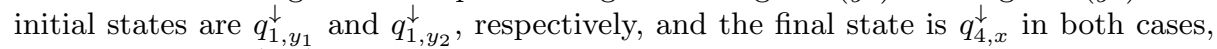
where the labels (1.4) of the rewriting rules are the ones from Example 1

The lemma below shows that these automata recognize exactly the labels of the minimal paths between two nodes:

Lemma 3. Let $\mathcal{T} \in \mathbb{T}_{\mathcal{R}}$ (b) be a rewriting tree and $w_{i} \in \operatorname{nodes}(\mathcal{T})$ be nodes labeled with the rules $\mathcal{T}\left(w_{i}\right)=\left(\mathrm{A}_{i}\left(x_{i, 1}, \ldots, x_{i, \#\left(\mathrm{~A}_{i}\right)}\right) \leftarrow \mathrm{b}_{i}\right)=\mathrm{r}_{i}$, for $i=1,2$. Then, for all $k_{i} \in\left[1, \#\left(\mathrm{~A}_{i}\right)\right], i=1,2$, the following are equivalent:

1. $x_{1, k_{1}}$ and $x_{2, k_{2}}$ are substituted by the same variable during the construction of $\mathfrak{C}_{[\mathcal{T}]}$ (Def. 4),

2. $\rho\left(w_{1}, w_{2}\right) \in \mathcal{L}\left(A_{\mathrm{r}_{1}, \mathrm{r}_{2}}^{x_{1}, x_{2}, x_{2}}\right)$.

The path automata $A_{\mathrm{r}_{1}, \mathrm{r}_{2}}^{x_{1}, x_{2, k_{2}}}$ are used to define the $P a t h_{\mathrm{r}_{1}, \mathrm{r}_{2}}^{z_{1}, z_{2}}$ formulæ:

$$
\begin{aligned}
\operatorname{Path}_{\mathrm{r}_{1}, \mathrm{r}_{2}}^{z_{1}, z_{2}}(x, y, \mathbf{U}) & \stackrel{\text { def }}{=} \exists \mathbf{Y} . \Delta_{A_{\mathrm{r}_{1}, \mathrm{r}_{2}}^{z_{1}, z_{2}}}(x, y, \mathbf{Y}) \wedge \Upsilon(\mathbf{Y}, \mathbf{U}) \\
\Upsilon(\mathbf{Y}, \mathbf{U}) & \stackrel{\text { def }}{=} \bigwedge_{d=\uparrow, \downarrow} \bigwedge_{i: \mathrm{r}_{i}=\left(\mathrm{A}^{\prime}\left(x_{1}, \ldots, x_{\#\left(\mathrm{~A}^{\prime}\right)}\right) \leftarrow \mathrm{b}^{\prime}\right)} \bigwedge_{z \in \mathrm{fv}\left(\mathrm{b}^{\prime}\right)} \forall x . Y_{\mathrm{r}, z}^{d}(x) \rightarrow U_{i}(x)
\end{aligned}
$$

The formula $\Upsilon(\mathbf{Y}, \mathbf{U})$ above states that all nodes labeled with a state $q_{\mathrm{r}, z}^{d}$ during the run must be also labeled with $r$ in the rewriting tree given as input to the path automaton. The lemma below proves that $\Phi\left(\mathbf{X}, \mathbf{X}^{\prime}, \mathbf{U}\right) \sqrt{12}$ is indeed a flow formula (Def. 5): 
Lemma 4. For each model $\nu$ of RTree $(\mathbf{U})$, we have $\nu \models \Phi\left(\mathbf{X}, \mathbf{X}^{\prime}, \mathbf{U}\right)$ if and only if $\sigma_{\nu}^{\mathbf{X}}={ }^{\bullet} t$ and $\sigma_{\nu}^{\mathbf{x}^{\prime}}=t^{\bullet}$ for some transition $t$ of $\mathcal{N}\left(\mathcal{T}_{\nu}^{\mathrm{U}}\right)$.

Together with Lemma 1 this ensures that the trap and mutex invariant of the parametric system described by $\mathrm{b}$ and $\mathcal{R}$ are defined by the $\operatorname{Trap}_{\operatorname{Inv}}{ }^{\Phi}(\mathbf{X}, \mathbf{U})$ and $\operatorname{MutexInv} v^{\Phi}(\mathbf{X}, \mathbf{U})$ formulæ, respectively. Hence a sufficient condition that proves a safety property of the parametric system described by $\mathrm{b}$ and $\mathcal{R}$ is the unsatisfiability of a WS $\kappa$ S formula, obtained from the syntax of $b$ and $\mathcal{R}$ :

Theorem 1. Let $\mathrm{b}$ be closed behavioral term, $\mathcal{R}$ be a rewriting system and $\mathcal{E}(\mathbf{X}, \mathbf{U})$ be a $\mathrm{WS} \kappa \mathrm{S}$ formula. The behavior $\mathcal{N}\left(\mathcal{T}_{\nu}^{\mathrm{U}}\right)$ is safe w.r.t. the set $\left\{\sigma_{\mu}^{\mathbf{X}} \mid \mu=\mathcal{E}(\mathbf{X}, \mathbf{U}), \mu(\mathbf{U})=\right.$ $\nu(\mathbf{U})\}$, for any valuation $\nu$, if the formula $R \operatorname{Tree}(\mathbf{U}) \wedge \operatorname{TrapInv}^{\Phi}(\mathbf{X}, \mathbf{U}) \wedge \operatorname{MutexInv}^{\Phi}(\mathbf{X}, \mathbf{U}) \wedge$ $\mathcal{E}(\mathbf{X}, \mathbf{U})$ is unsatisfiable.

In particular, we have experimented with error sets defined by the $\operatorname{DeadLock}^{\Phi}(\mathbf{X}, \mathbf{U})$ and CriticalSection ${ }^{\Xi}(\mathbf{X}, \mathbf{U})$ formulæ, for some critical section given by $\Xi \in \bigcup_{i=1}^{K} \mathcal{S}_{i}$.

\section{Experimental Evaluation}

We implemented the structural invariant synthesis in a prototype too ${ }^{6}$. Table 1 shows the results of checking deadlock freedom in all test cases and absence of critical section violations, for those test cases where a critical section was defined (otherwise marked $\mathrm{n} / \mathrm{a}$ ). The 2 nd column gives the number of states in the system, in the form $n_{1} \times \ldots \times n_{K}$, where $n_{i}$ is the number of states in the $i$-th component type and $K$ is the number of component types. The number of rewriting rules and interactions in the specification are given in the 3rd and 4th columns, respectively. The 5th and 7th columns report the results of the satisfiability check ( $\checkmark$ means that the formula is unsatisfiable and $\times$ means that a counterexample has been found, in which case safety could not be proved using our method) for deadlock freedom and absence of critical section violations, using the MonA v1.4-18 tool [11. The 6th and 8th columns show the total running times (in seconds) on an iMac $3,4 \mathrm{GHz}$ with $32 \mathrm{~GB}$ of RAM, respectively ( $\infty$ means that MonA has run out of memory). The 9 th column gives the branching degree $\kappa \in\{1,2\}$ of trees in the $\mathrm{WS} \kappa \mathrm{S}$ logic. Note that star and token ring systems require $\kappa=1$, whereas the tree-structured systems require $\kappa=2$.

The test cases we consider are grouped according to the architectural pattern. Token rings (Fig. 1 1 ) consist of instances of the same component type, such that the out port of a component is connected to the in port of the next component in the ring. Dining philosophers are special cases of token rings, consisting of alternating philosopher and fork instances. Stars consists of a single controller (master) sending requests and receiving replies from one or more slaves connected to it. Concerning trees, the tree-dfs example models a binary tree architecture traversed by a token in depth-first order, while tree-back-root and tree-linked-leaves(-generic) go beyond trees, modeling hierarchical systems with parent-children communication on top of which the nodes communicate with the root and the leaves are linked in a token-ring, respectively. These examples could not have been described using first order logic, as in 4. The verification problems considered could be solved in less than 1 second, with the exception of the critical section violations for the tree-linked-leaves(-generic) examples, that require mutex, in addition to trap invariants. In particular, in the examples marked with -generic, the initial state of the components is arbitrary. Consequently, all these examples violate the critical section initially.

\footnotetext{
6 https://github.com/raduiosif/rtab
} 
Table 1. Experimental Results

\begin{tabular}{|l|c|c|c|c|c|c|c|c|}
\hline Example & \#states & \#rules & $\begin{array}{c}\text { \#interaction } \\
\text { types }\end{array}$ & $\begin{array}{c}\text { deadlock } \\
\text { freedom }\end{array}$ & $\begin{array}{c}\text { time } \\
\text { (secs) }\end{array}$ & $\begin{array}{c}\text { critical } \\
\text { section }\end{array}$ & $\begin{array}{c}\text { time } \\
\text { (secs) }\end{array}$ \\
\hline \hline token-ring & $2 \times 2$ & 3 & 3 & $\checkmark$ & 0.66 & $\checkmark$ & 0.63 & 1 \\
token-ring-generic & $2 \times 2$ & 5 & 4 & $\checkmark$ & 0.75 & $\times$ & 0.72 & 1 \\
\hline sync-philo & $2 \times 2$ & 3 & 6 & $\checkmark$ & 0.69 & $\checkmark$ & 0.67 & 1 \\
alt-philo-sym & $3 \times 2$ & 3 & 9 & $\times$ & 0.75 & $\checkmark$ & 0.77 & 1 \\
alt-philo-asym & $3 \times 2$ & 3 & 9 & $\checkmark$ & 0.84 & $\checkmark$ & 0.78 & 1 \\
alt-philo-generic & $3 \times 2$ & 4 & 12 & $\checkmark$ & 0.91 & $\checkmark$ & 0.87 & 1 \\
\hline star & $2 \times 2$ & 3 & 4 & $\checkmark$ & 0.58 & $\mathrm{n} / \mathrm{a}$ & - & 1 \\
star-ring & $2 \times 3 \times 3$ & 3 & 9 & $\checkmark$ & 0.75 & $\checkmark$ & 0.76 & 1 \\
star-ring-generic & $2 \times 3 \times 3$ & 5 & 12 & $\checkmark$ & 0.84 & $\times$ & 0.88 & 1 \\
\hline tree-dfs & $2 \times 6 \times 2$ & 4 & 6 & $\checkmark$ & 0.70 & $\mathrm{n} / \mathrm{a}$ & - & 2 \\
tree-back-root & $2 \times 2$ & 3 & 5 & $\checkmark$ & 0.60 & $\mathrm{n} / \mathrm{a}$ & - & 2 \\
tree-linked-leaves & $2 \times 2 \times 4 \times 3$ & 4 & 10 & $\checkmark$ & 1.05 & $\checkmark$ & 1.21 & 2 \\
tree-linked-leaves-generic & $2 \times 2 \times 4 \times 3$ & 7 & 16 & $\checkmark$ & 1.31 & $\times$ & 1.73 & 2 \\
\hline
\end{tabular}

\section{Related Work}

Traditionally, verification of unbounded networks of parallel processes considers known architectural patterns, typically cliques or rings [106]. Because the price for decidability is drastic restrictions on the shape of architectures 3], more recent works propose practical semi-algorithms, e.g. regular model checking 131] or automata learning 7 . Here the architectural pattern is implicitly determined by the class of language recognizers: word automata encode pipelines or rings, whereas tree automata describe trees. A first attempt at specifying architectures by logic is the interaction logic of Konnov et al. 15], which is a combination of Presburger arithmetic with monadic uninterpreted function symbols, that can describe cliques, stars and rings. More structured architectures (pipelines and trees) can be described using a second-order extension [17]. As such, these interaction logics are undecidable and have no support for automated verification. Recently, interaction logics that support the verification of safety properties by structural invariant synthesis have been developed. These logics use fragments of first order logic with interpreted function symbols that implicitly determine the shape of the architecture [5:4].

\section{Conclusions and Future Work}

We present a formal language for the specification of distributed systems parameterized by the number of replicated components and by the shape of the coordinating architecture. The language uses inductive definitions to describe systems of unbounded size. We propose a verification method for safety properties based on the synthesis of structural invariants able to prove deadlock freedom for a number of non-trivial models.

\section{References}

1. Abdulla, P.A., Delzanno, G., Henda, N.B., Rezine, A.: Regular model checking without transducers (on efficient verification of parameterized systems). In: Grumberg, O., Huth, M. (eds.) Tools and Algorithms for the Construction and Analysis 
of Systems, 13th International Conference, TACAS 2007. LNCS, vol. 4424, pp. 721-736. Springer (2007)

2. Barrett, C.W., Shikanian, I., Tinelli, C.: An abstract decision procedure for a theory of inductive data types. J. Satisf. Boolean Model. Comput. 3(1-2), 21-46 (2007)

3. Bloem, R., Jacobs, S., Khalimov, A., Konnov, I., Rubin, S., Veith, H., Widder, J.: Decidability of Parameterized Verification. Synthesis Lectures on Distributed Computing Theory, Morgan \& Claypool Publishers (2015)

4. Bozga, M., Esparza, J., Iosif, R., Sifakis, J., Welzel, C.: Structural invariants for the verification of systems with parameterized architectures. In: Biere, A., Parker, D. (eds.) Tools and Algorithms for the Construction and Analysis of Systems - 26th International Conference, TACAS 2020. LNCS, vol. 12078, pp. 228-246. Springer (2020)

5. Bozga, M., Iosif, R., Sifakis, J.: Checking deadlock-freedom of parametric component-based systems. In: Vojnar, T., Zhang, L. (eds.) Tools and Algorithms for the Construction and Analysis of Systems - 25th International Conference, TACAS 2019. LNCS, vol. 11428, pp. 3-20. Springer (2019)

6. Browne, M., Clarke, E., Grumberg, O.: Reasoning about networks with many identical finite state processes. Information and Computation 81(1), 13 - 31 (1989)

7. Chen, Y., Hong, C., Lin, A.W., Rümmer, P.: Learning to prove safety over parameterised concurrent systems. In: Stewart, D., Weissenbacher, G. (eds.) 2017 Formal Methods in Computer Aided Design, FMCAD 2017. pp. 76-83. IEEE (2017)

8. Cousot, P., Cousot, R.: Systematic design of program analysis frameworks. In: Conference Record of the Sixth Annual ACM SIGPLAN-SIGACT Symposium on Principles of Programming Languages. pp. 269-282. ACM Press, New York, NY (1979)

9. Emerson, E.A., Namjoshi, K.S.: Reasoning about rings. In: Cytron, R.K., Lee, P. (eds.) Conference Record of POPL'95: 22nd ACM SIGPLAN-SIGACT Symposium on Principles of Programming Languages. pp. 85-94. ACM Press (1995)

10. German, S.M., Sistla, A.P.: Reasoning about systems with many processes. J. ACM 39(3), 675-735 (1992)

11. Henriksen, J.G., Jensen, J.L., Jørgensen, M.E., Klarlund, N., Paige, R., Rauhe, T., Sandholm, A.: Mona: Monadic second-order logic in practice. In: Brinksma, E., Cleaveland, R., Larsen, K.G., Margaria, T., Steffen, B. (eds.) Tools and Algorithms for Construction and Analysis of Systems, First International Workshop, TACAS '95. LNCS, vol. 1019, pp. 89-110. Springer (1995)

12. Iosif, R., Rogalewicz, A., Simácek, J.: The tree width of separation logic with recursive definitions. In: Bonacina, M.P. (ed.) Automated Deduction - CADE-24 - 24th International Conference on Automated Deduction. LNCS, vol. 7898, pp. 21-38. Springer (2013)

13. Kesten, Y., Maler, O., Marcus, M., Pnueli, A., Shahar, E.: Symbolic model checking with rich assertional languages. Theoretical Computer Science 256(1), 93-112 (2001)

14. Khoussainov, B., Nerode, A.: Automata Theory and its Applications. Springer (2001)

15. Konnov, I.V., Kotek, T., Wang, Q., Veith, H., Bliudze, S., Sifakis, J.: Parameterized systems in BIP: design and model checking. In: Desharnais, J., Jagadeesan, R. (eds.) 27th International Conference on Concurrency Theory, CONCUR 2016. LIPIcs, vol. 59, pp. 30:1-30:16. Schloss Dagstuhl - Leibniz-Zentrum für Informatik (2016) 
16. Kramer, J., Magee, J.: Analysing dynamic change in distributed software architectures. IEE Proceedings - Software 145(5), 146-154 (1998)

17. Mavridou, A., Baranov, E., Bliudze, S., Sifakis, J.: Configuration logics: Modeling architecture styles. J. Log. Algebr. Meth. Program. 86(1), 2-29 (2017)

18. Reynolds, J.C.: Separation logic: A logic for shared mutable data structures. In: 17th IEEE Symposium on Logic in Computer Science (LICS 2002). pp. 55-74. IEEE Computer Society (2002)

19. Thatcher, J., Wright, J.: Generalized finite automata theory with an application to a decision problem of second-order logic. Mathematical systems theory 2, 57-81 (2005) 